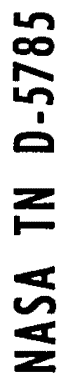

NASA IN D-5785

C. 1

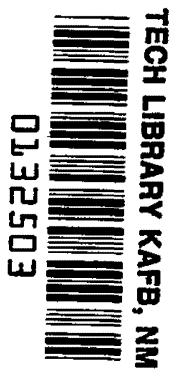

INAAN COPY: RETURN TO AFWL (WLOI)

KIRTIAND AFB, N MEA

\title{
GAUSS QUADRATURE RULES INVOLVING SOME NONCLASSICAL WEIGHT FUNCTIONS
}

by Paul F. Byrd and David C. Galant

Ames Research Center

Moffett Field, Calif. 94035

NATIONAL AERONAUTICS AND SPACE ADMINISTRATION - WASHINGTON, D. C. • MAY 1970 
1. Report No. NASA TN D-5785
2. Government Accession No.

4. Title and Subtitle GAUSS QUADRATURE RULES INVOLVING SOME NONCLASSICAL WEIGHT
FUNCTIONS

7. Author(s)

Paul F. Byrd and David C. Galant

9. Performing Organization Name and Address NASA Ames Research Center Moffett Field, Calif. 94035

12. Sponsaring Agency $\mathrm{Name}$ and Address National Aeronautics and Space Administration Washington, D. C. 20546

15. Supplementary Notes

16. Abstract

Gauss quadrature rules are given for numerically evaluating integrals on the interval $[0,1]$ for some weight functions of the form $w(t)=t^{\gamma}\left(1-t^{\alpha}\right) \beta$, where $\alpha$ is a positive integer $\geq 2$ and $\beta, \gamma>-1$. The nodes, weight coefficients and the parameters in the three-term recur'rence relation for the sequence of orthogonal polynomials associated with w(t) are tabulated to 25 significant figures for $N$-point rules with $\mathrm{N}=2,4,6,8,12,16$, and 24 . Numerical tables to $5 \mathrm{~S}$ are also
given for a coefficient in the expression of the error term.
3. Recipient's Catalog No.

5. Report Date

May 1970

6. Performing Organization Code

8. Performing Organization Report No. A-3553

10. Work Unit No.

129-04-04-02-00-21

11. Contraet or Gront No.

13. Type of Report and Period Covered Technical Note

14. Sponsoring Agency Code
17. Key Words Suggested by Author(s)

Gaussian quadrature Orthogonal polynomials Elliptic integrals

19. Security Classif. (of this report) Unclassified
18. Distribution Statement

Unclassified - Unlimited

20. Security Classif. (of this page)

Unclassified 
SYMBOLS . . . . . . . . . . . . . . . . . . . . . . . .

SUMMARY . . . . . . . . . . . . . . . . . . . . . . . . . . 1

INTRODUCTION . . . . . . . . . . . . . . . . . . . . . . . 1

GENERAL DISCUSSION OF CONSTRUCTION . . • . . . . . . . . . . . . . 4

Algebraic Approach . . . . . . . . . . . . . . . . . . . 5

Approach via Orthogonal Polynomials . . . . . . . . . . . . 5

NUMERICAL METHODS . . . . . . . . . . . . . . . . . . 6

Determination of the Moments . . . . . . . . . . . . . 6

Calculation of Parameters in the Three-Term Recurrence Relation ... 7

Calculation of the Nodes and Weight Coefficients . . . . . . . . 8

Calculation of the Error Coefficient . . . . . . . . . . . . . 10

Details of the Calculations . . . . . . . . . . . . . . 11

RESULTS . . . . . . . . . . . . . . . . . . . . . . . 11

APPENDIX A.- QR-ALGORITHM PROGRAM OF GOLUB AND WELSCH . • . . • . . . 12

REFERENCES . . . . . . . . . . . . . . . . . . . . 16

TABLES . . . . . . . . . . . . . . . . . . . . . 18 


\section{SYMBOLS}

$b_{n} \quad$ parameter in recurrence relation (see eq. (15))

$e_{k}^{(n)} \quad$ modified differences in Q-D scheme

$\mathrm{E}_{\mathrm{N}}$ error term of a quadrature formula (see eq. (2))

$g_{\mathrm{n}} \quad$ parameter in recurrence relation (see eq. (15))

$\mathrm{k}_{\mathrm{N}} \quad$ coefficient in the error term (see eq. (3))

$M_{n} \quad n t h$ moment of weight function $w(t)$ (see eq. (11))

$\mathrm{N}$ number of nodes in quadrature rule (see eq. (1))

$\mathrm{q}_{\mathrm{k}}^{(\mathrm{n})} \quad$ modified quotients in $\mathrm{Q}-\mathrm{D}$ scheme

S significant figures

$t_{j N} \quad j=1(1) N$, nodal points in quadrature formula (see eq. (1))

$w(t) \quad$ weight function

$W_{j N} \quad j=1(1) N$, weight coefficients in quadrature rule (see eq. (1))

$\Gamma(z) \quad$ gamma function of argument $z$

$\phi_{k}(t)$ orthogonal polynomial of degree $k$ (see eq. (15)) 
GAUSS QUADRATURE RULES INVOLVING SOME NONCLASSICAL

WEIGHT FUNCTIONS

Paul F. Byrd and David C. Galant

Ames Research Center

SUMMARY

Gauss quadrature rules are given for numerically evaluating integrals on the interval $[0,1]$ for some weight functions of the form $w(t)=t \gamma\left(1-t^{\alpha}\right)^{\beta}$, where $\alpha$ is a positive integer $\geqslant 2$ and $\beta, \gamma>-1$. The nodes, weight coefficients, and the parameters in the three-term recurrence relation for the sequence of orthogonal polynomials associated with $w(t)$ are tabulated to 25 significant figures for $N$-point rules with $N=2,4,6,8,12,16$, and 24 . Numerical tables to $5 \mathrm{~S}$ are also given for a coefficient in the expression of the error term.

\section{INTRODUCTION}

A wide variety of problems requiring numerical approximations to specific definite integrals arise very often in practice, usually as an intermediate step in a more extensive computation. There are many methods for approximating such integrals, but among all quadrature formulas of the form

$$
I=\int_{a}^{b} g(t) d t \equiv \int_{a}^{b} w(t) f(t) d t \simeq \sum_{j=1}^{N} w_{j N} f\left(t_{j N}\right)
$$

only the weighted gaussian types have all their parameters chosen to maximize performance. For these formulas, the nodes $t_{j N}$ and the coefficients $W_{j N}$ are uniquely determined so that all approximations to the given integral are exact for arbitrary polynomials $f$ of the highest possible degree, $2 \mathrm{~N}-1$. If the function $f$ is other than a polynomial of degree $\leqslant 2 N-1$, the integration formulas (1) have an error term given (e.g., see ref. 1) by

where

$$
E_{N}=\frac{k_{N}}{(2 N) !} f^{(2 N)}(\tau), \quad a<\tau<b
$$

$$
k_{N}=\int_{a}^{b} w(t) \phi_{N}^{2}(t) d t
$$

with $\phi_{\mathrm{N}}$ being the associated Nth-degree orthogonal polynomial. 
The literature concerning weighted gaussian formulas is very large, both classical and recent (refs. 2, 3, and 4). Widespread use of high-speed computers, with emphasis on economizing the number of operations required in computations, has resulted in considerable renewed interest in the power of the gaussian technique; gauss quadrature rules are being tabulated and used with increasing frequency. Very accurate rules, for example, were recently tabulated in references 1 and 5 for cases associated with the classical orthogonal polynomials of Legendre, Laguerre, Chebyshev, Jacobi, and Hermite. Recent numerical tables to many significant figures have also been given for some nonclassical cases such as a weight function on $[0,1]$ having logarithmic singularities (ref. 6) and the weight function $e^{-x^{2}}$ on the interval $[0, \infty]$ (ref. 7).

Weighted gaussian integration formulas are excellent for large classes of functions arising in practice. They are particularly useful when a complete integrand $g(t)$ is factorable into two functions $f(t)$ and $w(t)$, where $f$ is well approximated by a polynomial, with the nonnegative weight function $w$ having a singularity or a singular derivative at one or both ends of the interval. Since neither the error term (2) nor the sum in the approximations (1) directly involves the weight function $w$, once the quadrature rules are constructed and programmed on a computer, they can be repeatedly applied to integrals of the form (1) with different functions $f(t)$ and the same $w(t)$. There are several other advantages of gauss-type formulas: (a) since all the coefficients $W_{j N}$ turn out to be positive, the rules have good roundoff properties; (b) the sequence of formulas (1) for $N=1,2$, . . . will always converge to the value of the Riemann integral I whenever this integral exists; (c) the rules have more favorable error terms and generally achieve higher accuracy with the use of fewer nodes than Newton-Cotes methods.

This report presents tabulated gaussian quadrature formulas for

$$
I(\alpha, \beta, \gamma ; f)=\int_{0}^{1} w(\alpha, \beta, \gamma ; t) f(t) d t \simeq \sum_{j=1}^{N} w_{j N} f\left(t_{j N}\right)
$$

where the weight function $w$ is of the form

$$
w(\alpha, \beta, \gamma ; t) \equiv w(t)=t^{\gamma}\left(1-t^{\alpha}\right)^{\beta}
$$

with $\alpha$ as a positive integer $\geqslant 2 ; \beta, \gamma>-1$; and $w$ or its derivative singular at one or both ends of the interval. For the special case $\alpha=1$, the approximate relation (4) becomes the classical Gauss-Jacobi formula

$$
\int_{0}^{1} t^{\gamma}(1-t)^{\beta} f(t) d t \simeq \sum_{j=1}^{N} w_{j N} f\left(t_{j N}\right)
$$


Krylov, Lugin, and Janovic (ref. 8) recently gave these quadrature rules to eight significant figures for $\beta, \gamma=-0.9(0.1) 3.0, \beta \leqslant \gamma$ and $N=1(1) 8$.

We shall consider various cases where the parameters $(\alpha, \beta, \gamma)$ are

$$
\begin{aligned}
& \alpha=2,3,4,6,8 \\
& \beta=-3 / 4,-2 / 3,-1 / 2,1 / 2 \\
& \gamma=-1 / 2,0,1 / 2
\end{aligned}
$$

One of these, for example, is the integral

$$
I(3,-1 / 2,0 ; f)=\int_{0}^{1} \frac{f(t)}{\sqrt{1-t^{3}}} d t
$$

which, when $f(t)$ is simply a rational function, may be reduced by a substitution involving Jacobian elliptic functions to a combination of three standard tabulated elliptic integrals (ref. 9). A similar statement applies to integrals $I(\alpha, \beta, \gamma ; f)$ with regard to the other values $\alpha, \beta, \gamma$ given in equations (7).

If $f$ is not a rational function (e.g., $e^{-c t}$ ), none of the integrals $\mathrm{I}(\alpha, \beta, \gamma)$ are expressible in finite terms of known tabulated functions for the chosen parameters $(\alpha, \beta, \gamma)$, and the only recourse is numerical approximation via some quadrature formula. Even when such integrals can be written as combinations of standard elliptic integrals etc., it is probably more efficient to use quadrature rules having the highest degree of algebraic precision to obtain answers. Integrals with our weight functions $w(t)$ uccur sufficiently frequently in practice to warrant the construction of such rules.

Numerical tables for $N$-point gaussian rules $\left\{w_{j N}, t_{j N}\right\}$ with $\mathrm{N}=2,4,6,8,12,16$, and 24 are given to 25 significant figures for 12 special cases of $I(\alpha, \beta, \gamma ; f)$. These are for

$$
(\alpha, \beta, \gamma)=\left\{\begin{array}{lllll}
(3,-1 / 2,0) & (3,1 / 2,0) & (4,-1 / 2,0) & (4,1 / 2,0) \\
(6,-1 / 2,0) & (6,1 / 2,0) & (8,-1 / 2,0) & (8,1 / 2,0) \\
(3,-1 / 2,-1 / 2) & (3,1 / 2,1 / 2) & (2,-3 / 4,0) & (2,-2 / 3,0)
\end{array}\right\}
$$

The coefficients $b_{n}, g_{n}$ in the three-term recursion relation (15) for the sequence of monic orthogonal polynomials corresponding to $w(t)$ are also tabulated to $25 \mathrm{~S}$ for $\mathrm{N}=1$ (1) 26. Since the error coefficients $\mathrm{k}_{\mathrm{N}}$ in the remainder term (2) may be useful when a bound on the $2 \mathrm{Nth}$ derivative can be estimated, we give tabulations of them to 5 S. 
Little difficulty is entailed in the actual construction of gauss-type quadrature formulas relative to classical weight functions where the threeterm recurrence relation for the associated orthogonal polynomials are known explicitly. This is not generally true for nonclassical $w(t)$ such as ours where the sequence of related unknown orthogonal polynomials must be generated using the moments of $w$.

\section{GENERAL DISCUSSION OF CONSTRUCTION}

For a given nonnegative weight function $w(t)$ on $[0,1]$, the problem of constructing the $\mathrm{N}$-point gaussian quadrature

$$
G_{N}(f)=\sum_{j=1}^{N} w_{j N} f\left(t_{j N}\right)=\int_{0}^{1} w(t) f(t)-E_{N}(f), \quad(N=1,2, \ldots . .)
$$

consists in determining the nodes $t_{j N}$ and the weight coefficients $W_{j N}(2 \mathrm{~N}$ parameters in all) so that the error $E_{N}(f)=0$ whenever $f$ is a polynomial of degree $n \leqslant 2 N-1$. There are essentially only two approaches to the determination of these unknown parameters, and they sha11 be discussed briefly.

Before proceeding, however, we wish to emphasize in the strongest possible terms the numerical difficulties inherent in the construction of the quadrature rule (10) from the moments

$$
M_{k}=\int_{0}^{1} t^{k} w(t) d t, \quad k=0(1) 2 N-1
$$

Gautschi (ref. 4) has shown that an approximate upper bound to the relative, asymptotic condition number of this problem is

$$
C_{N}=\frac{(17+12 \sqrt{2})^{N}}{64 N^{2}}
$$

This means that the computation is extremely il1-conditioned numerically, and that the number of significant figures lost in determining the rule (10) using the moments (11) is approximately

$$
S_{N}=\log _{10} C_{N}=1.53 N-2 \log _{10}(8 N)
$$


Thus, if one wants to have the nodes and weights of $\mathrm{G}_{\mathrm{N}}$ accurate to $k$ significant figures, then he must work with more than

$$
k+1.53 N-2 \log _{10}(8 N)
$$

significant figures. For example, to obtain a 10-point quadrature rule with nodes and weights accurate to 10 significant digits (i.e., $N=k=10$ ), one must work with more than 22 significant digits at a certain stage in the calculation of the nodes and weights.

\section{Algebraic Approach}

If the nodes $t_{j N}$ and the weight coefficients $w_{j N}$ in equation (10) are chosen so that the formula is exact for all polynomials $f(t)$ of degree $\mathrm{n} \leqslant 2 \mathrm{~N}-1$, then it is sufficient to choose the formula to be exact for the monomials $t^{k}, k=0(1) 2 \mathrm{~N}-1$, so that one has the system

$$
\sum_{j=1}^{N} w_{j N} t_{j N}^{k}=M_{k}, \quad k=0(1) 2 N-1
$$

of $2 \mathrm{~N}$ nonlinear algebraic equations in $2 \mathrm{~N}$ unknowns to solve, where $M_{k}$ are given by equation (11). The drawback to this formulation of determining the quadrature rule from the moments is that any attempt at a direct solution requires the highly precise arithmetic indicated by equation (13) at every step of the calculations. For this reason, the algebraic approach is to be rejected except for very small $\mathrm{N}$.

\section{Approach Via Orthogonal Polynomials}

Orthogonal polynomials play an important role in gaussian quadratures. In fact, it can easily be shown that if the nodal points $t_{j N}$ in formula (10) are selected to be the roots of the $N$ th-degree polynomial $\phi_{N}(t)$ of the sequence of orthogonal polynomials $\left\{\phi_{n}(t)\right\}$ associated with the given weight function $w(t)$, then the formula is exact for any polynomial $f(t)$ of degree $\leqslant 2 N-1$; the converse of this is also true (e.g., see ref. 10).

The orthogonal system of polynomials associated with $w(t)$ in $(a, b)$ is unique and any three consecutive polynomials satisfy the recurrence relation

$$
\phi_{n}(t)=\left(t-b_{n}\right) \phi_{n-1}(t)-g_{n} \phi_{n-2}(t), \quad n=1(1) N
$$

with $\phi_{-1}(t)=0, \phi_{0}(t)=1$, and 


$$
\left.\begin{array}{rl}
b_{n}= & \frac{\int_{0}^{1} t w(t) \phi_{n-1}^{2}(t) d t}{\int_{0}^{1} w(t) \phi_{n-1}^{2}(t) d t} \\
g_{n+1}= & \int_{0}^{1} w(t) \phi_{n}^{2}(t) d t \\
\int_{0}^{1} w(t) \phi_{n-1}^{2}(t) d t
\end{array}\right\}
$$

The usual approach in the construction of gaussian rules is based on the use of relation (15) and the moments to generate the sequence of polynomials $\left\{\phi_{k}(t)\right\}$. After these are calculated, one solves, in some way, for the roots $t_{j N}$ of $\phi_{N}(t)$, and then finds the weight coefficients $W_{j N}$ by other means. However, any procedure that relies on the moments is essentially equivalent to solving the basic algebraic system (14) and is highly unstable numerically.

The advantage of the approach via orthogonal polynomials, as we shal1 see, is that the ill-conditioned nature of the problem is encountered in the determination of the three-term recurrence relation from the moments, but at no point in the determination of the nodes and weights of the quadrature rule. Since the calculation of $b_{n}, g_{n}, n=1(1) N$, needs less than $4 N^{2}$ arithmetic operations, the amount of multiply precise arithmetic is small and manageable, thus making this approach very tractable in comparison to the algebraic approach.

\section{NUMERICAL METHODS}

The numerical construction of our gaussian rules proceeds in three steps: (1) Determine, to sufficient accuracy, the moments $M_{k}$ for $k=0(1) 2 N-1$; (2) using the moments, calculate the parameters $b_{k}, g_{k}, k=1(1) N$, in the three-term recurrence relation (15) for the first $N$ orthogonal polynomials associated with the weight function $w(t)$ on $(0,1)$; (3) determine the quadrature nodes $t_{j N}$ and the weight coefficients $w_{j N}$ for $j=1(1) N$. The three steps will be discussed in that order.

\section{Determination of the Moments}

The moments for the weight function of interest are

$$
M_{n}(\alpha, \beta, \gamma) \equiv M_{n}=\int_{0}^{1} t^{n} w(t) d t=\int_{0}^{1} t^{n+\gamma}\left(1-t^{\alpha}\right)^{\beta} d t
$$

for $n=0(1) 2 N-1$. If we let $z=t^{\alpha}$, equation (17) becomes 


$$
M_{n}=\frac{1}{\alpha} \int_{0}^{1} z^{(n+\gamma+1) /(\alpha-1)}(1-z)^{(1+\beta)-1} d z, \quad(\alpha>0 ; \beta, \gamma>-1)
$$

or

$$
M_{n}=\frac{1}{\alpha} B\left(n+\frac{\gamma+1}{\alpha}, 1+\beta\right)=\frac{\Gamma(n+\gamma+1 / \alpha) \Gamma(1+\beta)}{\alpha \Gamma[1+\beta+(n+\gamma+1 / \alpha)]}
$$

where $B$ and $\Gamma$ are the beta and gamma functions, respectively. Using the well-known recurrence relation for the gamma function, we have the recursion relation

$$
M_{k}=1+[\alpha(1+\beta) /(k+\gamma-\alpha+1)], \quad k=\alpha(1) 2 N-1
$$

so that al1 the moments can be generated from $M_{0}, M_{1}, \ldots ., M_{\alpha-1}$.

\section{Calculation of Parameters in the Three-Term Recurrence Relation}

There are several methods of calculating the three-term recurrence relation from the moments in the general case (refs. 1 and 11). However, for several reasons, mainly because of the volume of arithmetic involved, we have chosen a more specialized approach. We use the quotient-difference algorithm (ref. 12) to obtain the three-term recurrence relation parameters from the moments. This method will always work when the interval of integration does not have the origin as an interior point. The procedure is briefly as follows: Define

$$
e_{0}^{(j)}=0, \quad q_{1}^{(j)}=\frac{M_{j+1}}{M_{j}}, \quad j=0(1) 2 N-2
$$

Generate the rest of the table

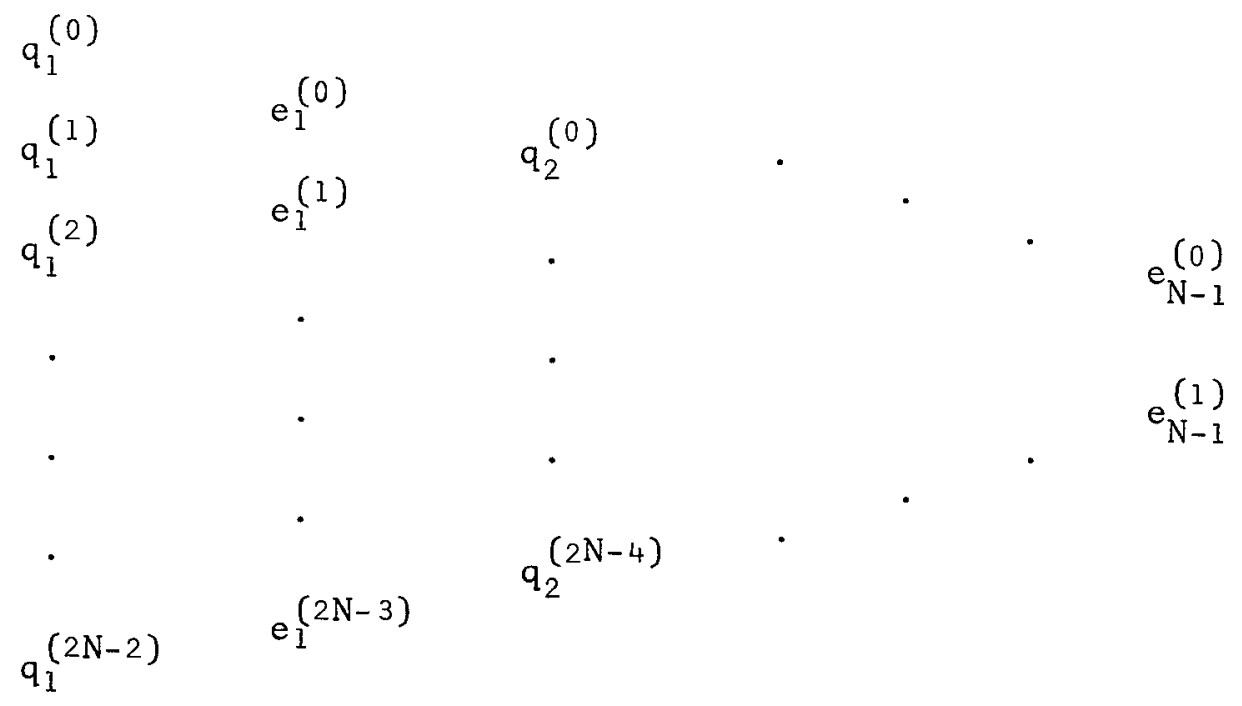


using the rhombus rules

$$
\left.\begin{array}{l}
e_{k}^{(n)}=e_{k-1}^{(n+1)}+\left[q_{k}^{(n+1)}-q_{k}^{(n)}\right], \quad n=0(1) 2(N-k)-1 \\
q_{k+1}^{(n)}=\frac{q_{k}^{(n+1)} e_{k}^{(n+1)}}{e_{k}^{(n)}}, \quad n=0(1) 2(N-k)
\end{array}\right\}
$$

with $k=I(1) N-1$. The parameters for relation (15) are then expressed by

with $g_{I}=0$.

$$
\left.\begin{array}{ll}
b_{n}=q_{n}^{(0)}+e_{n-1}^{(0)}, & n=1(1) N \\
g_{n}=q_{n-1}^{(0)} e_{n-1}^{(0)}, & n=2(1) N
\end{array}\right\}
$$

\section{Calculation of the Nodes and Weight Coefficients}

The three-term recurrence relation (15) for the first $N$ orthogonal polynomials can be written (ref. 13) as the maxtrix equation

$$
t \Phi(t)=A \Phi(t)+B \phi_{N}(t)
$$

where

$$
\Phi(t)=\left[\begin{array}{c}
\phi_{0}(t) \\
\phi_{1}(t) \\
\phi_{2}(t) \\
\cdot \\
\cdot \\
\phi_{N-2}(t) \\
\phi_{N-1}(t)
\end{array}\right] \quad B=\left[\begin{array}{c}
0 \\
0 \\
0 \\
\cdot \\
\cdot \\
0 \\
1
\end{array}\right]
$$

and 


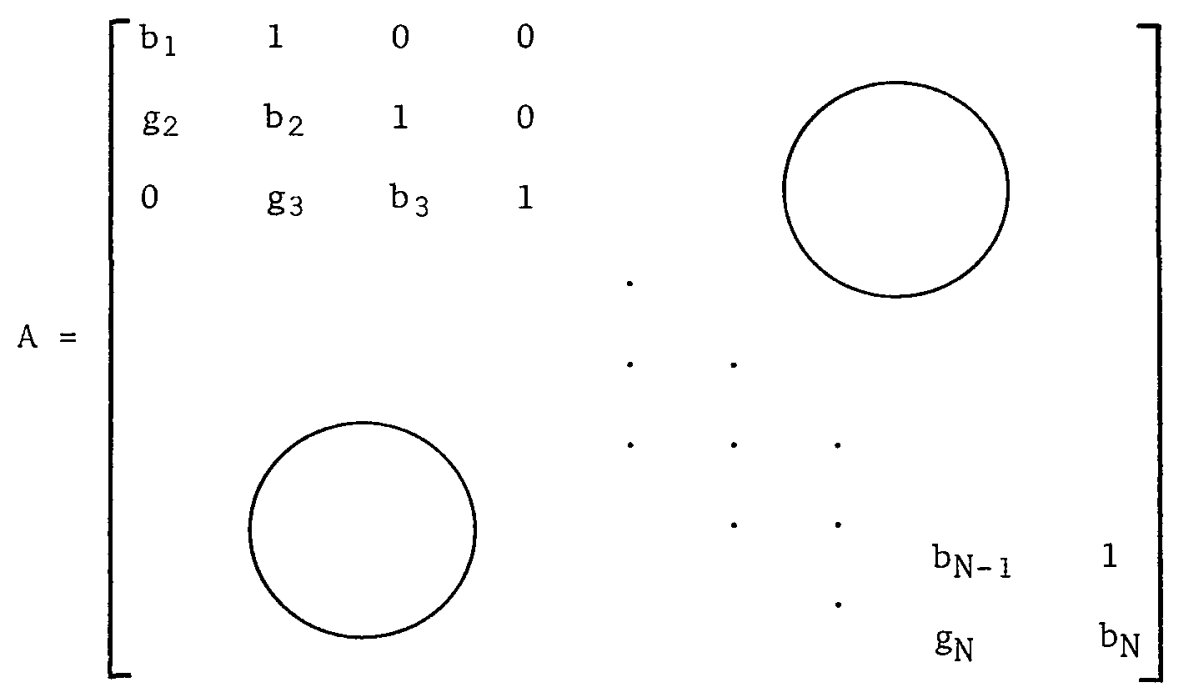

(25)

From equation (23) it is immediately seen that the roots of $\phi_{N}(t)$, which are the nodes of the quadrature rule, are also the eigenvalues of the matrix $A$. Applying a diagonal similarity transformation to the matrix (25) yields the symmetric Jacobi matrix

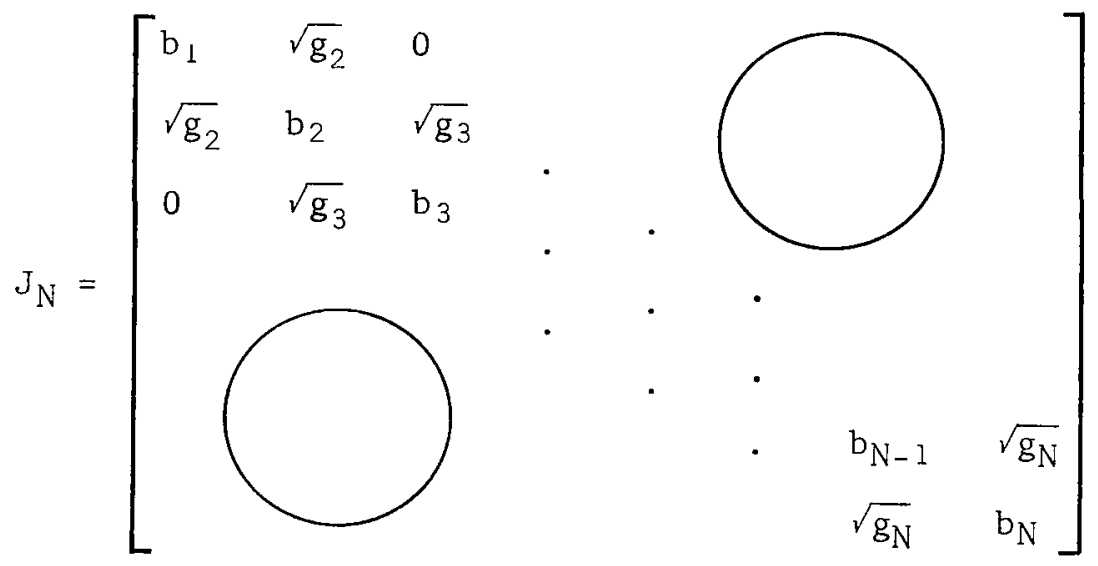

which has the same eigenvalues as A. In reference 13, it is further shown that if $Q_{1, j}$ is the first component of the orthonormal eigenvector of $J_{N}$ associated with the eigenvalue $t_{j N}$, then

$$
W_{j N}=Q_{1, j}^{2} M_{0}
$$

Hence, both the nodes and the weight coefficients of the quadrature rule (10) can be calculated from the eigensystem of the symmetric tridiagonal matrix (26). The version of the very fast numerically stable QR algorithm (ref. 14) given in reference 12 to accomplish this computation was used. 


\section{Calculation of the Error Coefficient}

The coefficient of the error term (2) can be obtained from the $g_{n}$. From equations (16) and equation (3) it immediately follows that

$$
k_{N}=M_{0} \prod_{i=1}^{N} g_{i+1}
$$

These constants are useful in obtaining exror bounds if one has an estimate of $\max |f(2 N)(\tau)|$.

$0<\tau<1$

\section{Details of the Calculations}

From the formulation of the calculation of quadrature rule (1) from the three-term recurrence relation parameters (16) as the determination of the eigensystem of the symmetric tridiagonal matrix (26) with no multiple eigenvalues, it can be shown (ref. 15) that once the three-term recurrence relation is known, no error is incurred except for round-off error. Therefore, all of the ill-conditioning of the calculation of the quadrature rule from the moments must occur in the determination of the recurrence relation. With this fact in mind along with the aim of obtaining a 24-point quadrature rule with nodes and weights accurate to 25 significant figures, the following calculations were carried out:

(a) The moments $M_{n}, n=0(1) 51$, were calculated from the recurrence relation (19) in 70-digit floating-point arithmetic. The first $\alpha$ moments were calculated using appropriate values of the gamma function accurate to 65 significant figures. These values were obtained by the method given in reference 16 .

(b) The coefficients $b_{n}, g_{n}, n=1(1) 26$, were obtained from the moments via the quotient-difference algorithm. This computation was also done in 70-digit floating-point arithmetic. Using Gautschi's estimate of the condition number (which is remarkably accurate) we find that $b_{25}, g_{25}$ had

$$
k=65-1.53(25)+2 \log _{10}(200) \simeq 30
$$

significant figures.

(c) The eigenvalues and the first components of the orthonorinal eigenvectors of the matrix $J_{N}$ (eq. (26)) were calculated, using a version of the algorithm Gaussquadrule given in reference 12, for $N=2(2) 8(4) 16,24$. These computations were done using 30-digit floating-point arithmetic.

In order to check these quadrature rules, the identities

$$
\sum_{j=1}^{N} w_{j N}=M_{0}, \quad \sum_{j=1}^{N} b_{j}=\sum_{j=1}^{N} t_{j N}
$$


were used. It is our experience that, for quadrature rules on a finite interval, these are sufficient to determine the computed accuracy of the nodes and weight coefficients. In no instance was the disagreement more than a relative error of $10^{-28}$.

\section{RESULTS}

In tables 1 through 12 , the nodes and weight coefficients of the $\mathrm{N}$-point quadrature rules for $\mathrm{N}=2(2) 8(4) 16,24$ are given for the 12 previously enumerated weight functions. Each of the nodes and weight coefficients is rounded to 25S. In table 13 the error constants $\mathrm{k}_{\mathrm{N}}$, for the same values of $\mathrm{N}$, are given to 5 significant figures.

Tables 14 through 25 are tabulations of $M_{0}$ and of $b_{n}, g_{n}, n=1(1) 26$ of the recurrence relation for the monic orthogonal polynomials associated with the enumerated weight function $w(t)$. These tabulations are also rounded to 25 significant figures.

Appendix $A$ is a listing of a double precision version of the algorithm used to obtain the quadrature rules. This program can be used to determine practical accuracy quadrature rules from the three-term recurrence relation parameters. In particular, it can be employed to find quadrature formulas not tabulated in this report.

The parameters associated with each table are summarized by:

\begin{tabular}{|r|c|c|c|}
\hline Table & $\alpha$ & $\beta$ & $\gamma$ \\
\hline 1,14 & 3 & $-1 / 2$ & 0 \\
2,15 & 3 & $1 / 2$ & 0 \\
3,16 & 4 & $-1 / 2$ & 0 \\
4,17 & 4 & $1 / 2$ & 0 \\
5,18 & 6 & $-1 / 2$ & 0 \\
6,19 & 6 & $1 / 2$ & 0 \\
7,20 & 8 & $-1 / 2$ & 0 \\
8,21 & 8 & $1 / 2$ & 0 \\
9,22 & 3 & $-1 / 2$ & $-1 / 2$ \\
10,23 & 3 & $1 / 2$ & $1 / 2$ \\
11,24 & 2 & $-3 / 4$ & 0 \\
12,25 & 2 & $-2 / 3$ & 0 \\
\hline
\end{tabular}

In the tables, $a \pm b$ denotes $a \cdot 10^{ \pm b}$; for instance, $0.17-6$ means $0.17 \cdot 10^{-6}$.

\section{Ames Research Center}

National Aeronautics and Space Administration Moffett Field, Calif. 94035, January 19, 1970 
QR ALGORITHM PROGRAM OF GOLUB AND WELSCH

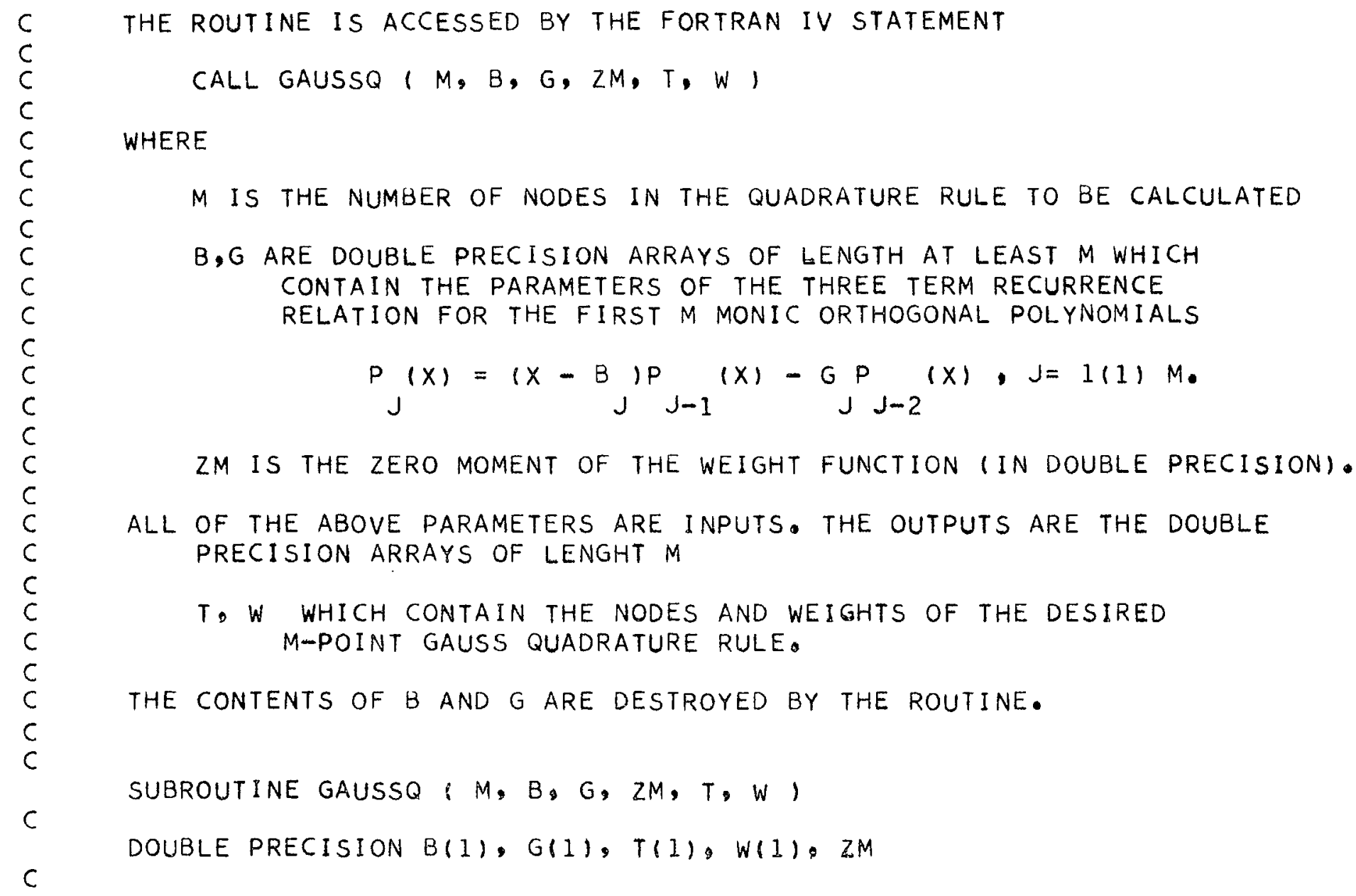




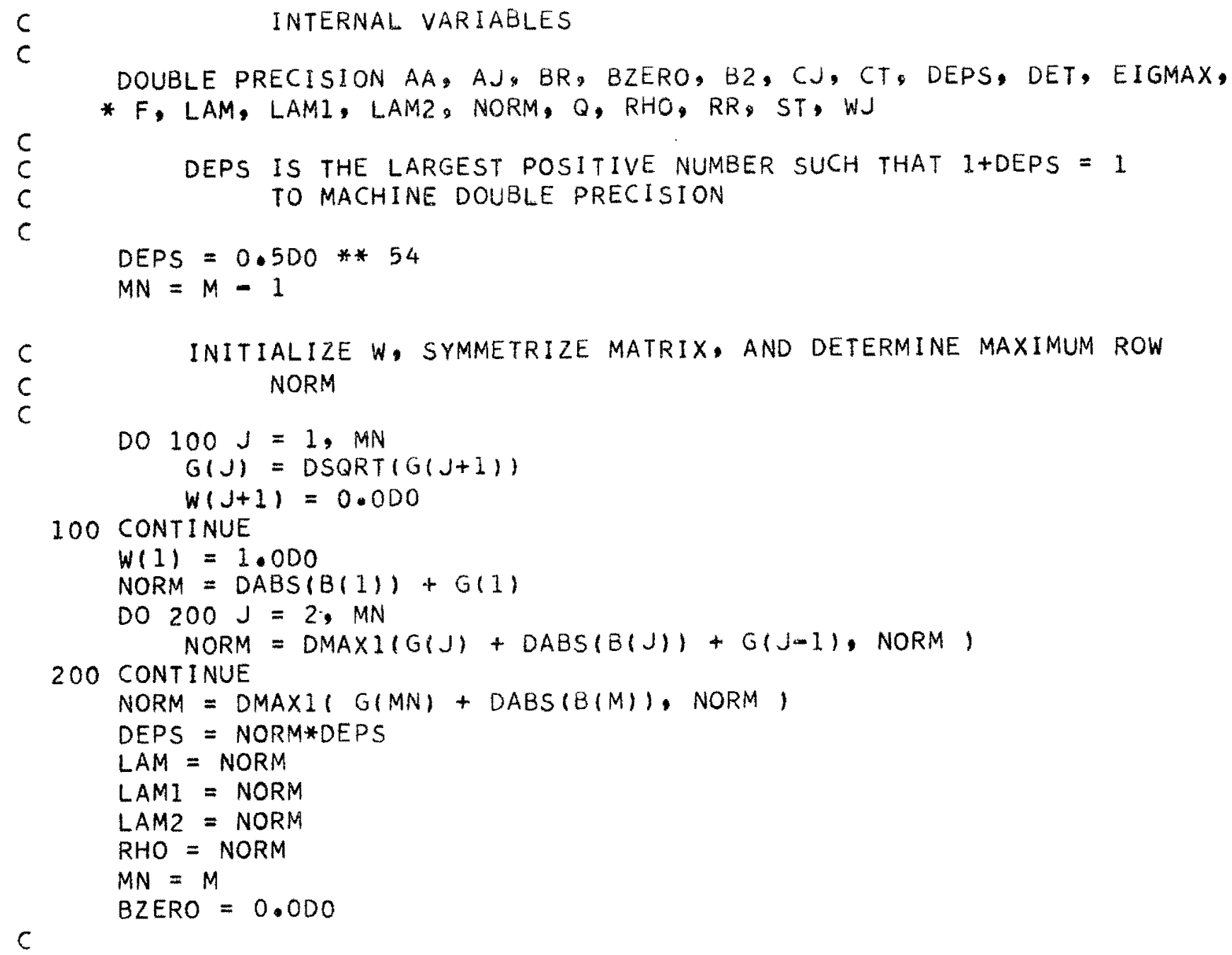

DOUBLE PRECISION AA, AJ, BR, BZERO, B2, CJ, CT, DEPS, DET, EIGMAX,

$c$

c 


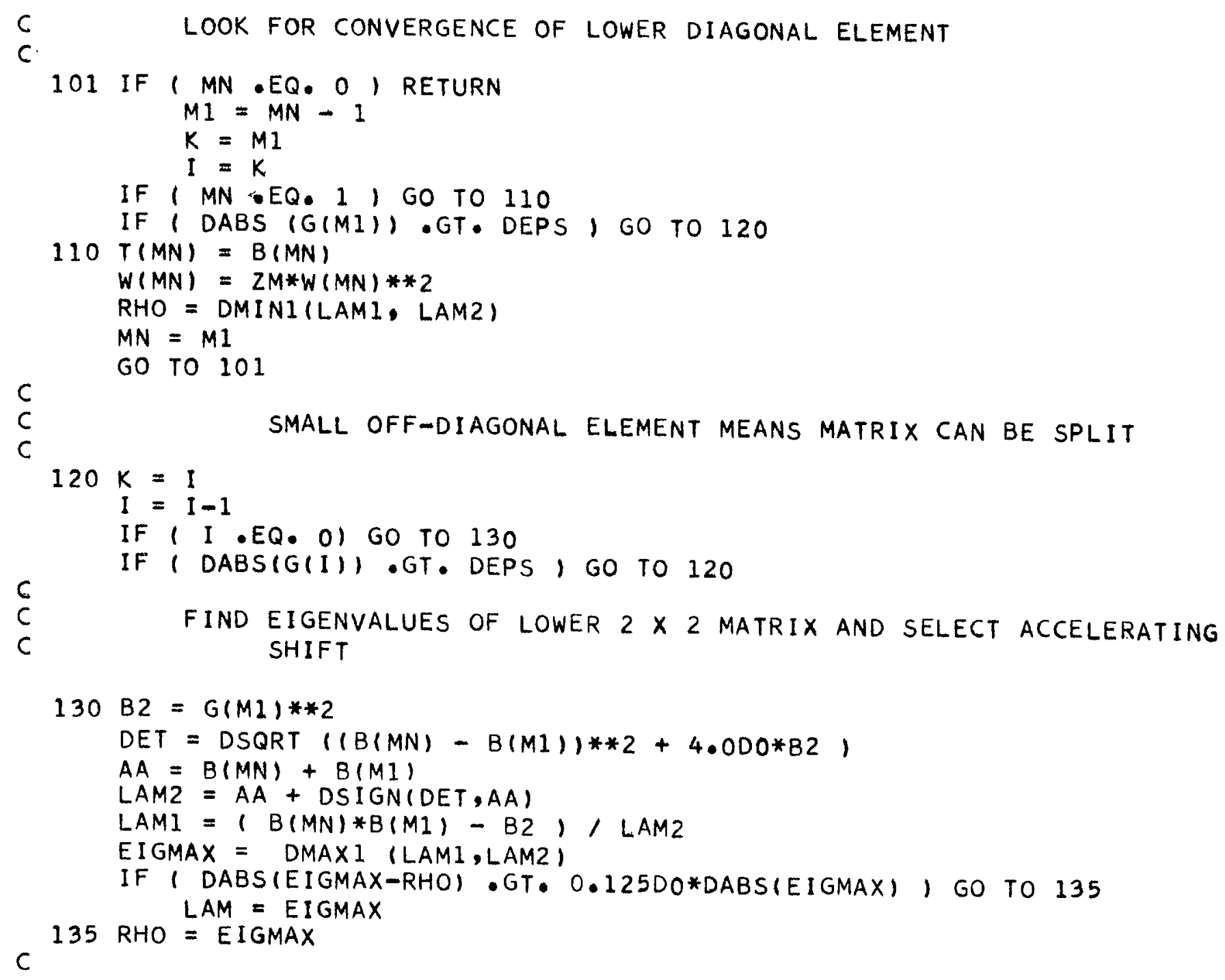




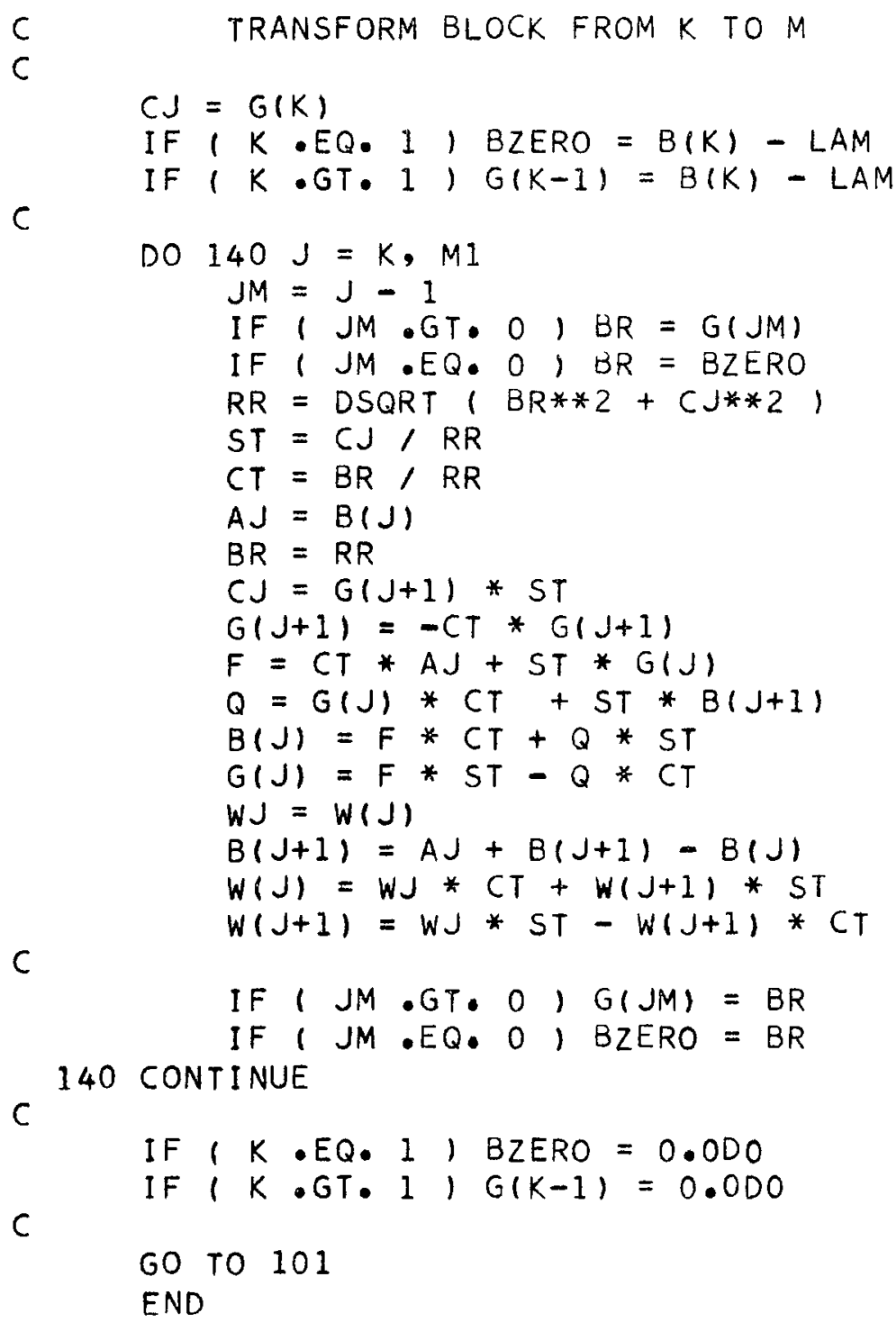




\section{REFERENCES}

1. Stroud, A.; and Secrest, D.: Gaussian Quadrature Formulas. PrenticeHa11, Englewood Cliffs, N. J., 1966.

2. Gauss, C. F.: Methodus Nova Integralium Valores per Approximationen Inveniendi. Comment. Soc. Regiae Sci. Gottingensis Recentiores, vol. 3, p. 1816; Werke, vol. 3, pp. 163-196.

3. Stroud, A. H.: A Bibliography on Approximate Integration. Mathematical Tables and Other Aids to Computations. Math. Comput., vol. 15, 1961, pp. 52-80.

4. Gautschi, W.: Construction of Gauss-Christoffel Quadrature Formulas. Math. Comput., vol. 22, no. 102, April 1968, pp. 251-270.

5. Shao, T. S.; Chen, T. C.; and Frank, R. M.: Tables of Zeros and Gaussian Weights of Certain Associated Laguerre Polynomials and the Related Generalized Hermite Polynomials. Math. Comput., vol. 18, no. 88 , Oct. 1964, pp. 598-616.

6. Krylov, V. I.; and Pal'cev, A. A.: Numerical Integration of Functions Having Logarithmic and Power Singularities. Vesci Akad. Navuk BSSR Ser. Fiz.-Téhn. Navuk, no. 1, 1963, pp. 14-23 (Russian).

7. Galant, D.: Gauss Quadrature Rules for the Evaluation of $(2 / \sqrt{\pi})$ $\int_{0}^{\infty} \exp \left(-x^{2}\right) f(x) d x$. Math. Comput., vol. 23, no. 107, July 1969, p. 674.

8. Krylov, V. I.; Lugin, V. V.; and Janovic, L. A.: Tables for Numerical Integration of Functions With Power Singularities $\int^{1} x^{\beta}(1-x)^{\alpha} f(x) d x$. Izdat. Akad. Nauk. Belorussk. SSR, Minsk, 1963 (Russian).

9. Byrd, P. F.; and Friedman, M. D.: Handbook of Elliptic Integrals for Engineers and Physicists. Springer-Verlag, Berlin, Germany, 1954.

10. Davis, P. J.; and Rabinowitz, P.: Numerical Integration. Blaisde11 Pub. Co., Waltham, Mass., 1967.

11. Golub, G. H.; and Welsch, J. H.: Calculation of Gauss Quadrature Rules. Math. Comput., vo1. 23, no. 106, April 1969, pp. 221-230.

12. Henrici, P.: The Quotient-Difference Algorithm. Appl. Math. Ser. 49, Nat1. Bur. Standards, 1958, pp. 23-46.

13. Wilf, H.: Mathematics for the Physical Sciences. John Wiley and Sons, Inc., N. Y., 1962 . 
14. Francis, J. G. F.: The Q-R Transformation: A Unitary Analogue to the L-R Transformation. I, II. Comput. J., vol. 4, 1961, pp. 265-271; $332-345$.

15. Wilkinson, J. H.: The Algebraic Eigenvalue Problem. Oxford Univ. Press (Clarendon), London and N. Y., 1965.

16. Galant, D. C.; and Byrd, P. F.: High Accuracy Gamma Function Values for Some Rational Arguments. Math. Comput., vo1. 22, no. 104, Oct. 1968, pp. 885-888. 
.235650262817864226489023900 $.8712343337313635582217330+0$

$.5652422304414819460830784+0$ $.8369392748839723150919407+0$

$N=4$

$7337729738653181679464650-1$ $3501778318150437158234797+0$ $7105740027251409197101383+0$ 964213000520037953952780940

1840804095652859416343037 t0 $.3552780729357526979745190+0$ $420168147000615316641873+0$ $4388668081243540899020090+1$

350445704ECR 4343418449311-1 $1760429136520695590901530+0$ . 396451 5290272939727325090+0 $.6449294589447569740660749+0$ $8395059162165180624249422+0$ $9836370121825846374622039+0$

88S331 405C394839795691733-1 . $1883153151023423552937622+0$ .2523027875990548598314225 .2829312063589059800454765 2976009070014966007373740 7960967487596960640100666+0

$2041981505827257341810990-1$ $1046136300575156432957783+0$ 2443710169441164617315762+口 $.4210426148229107978985786+0$ .61 Ce23946579530890947766840 7846479140515148192768200 .9182694706544585845538558+0 $9906767463370753336401726+0$

$5205969893370611545022507-1$ .1145639325324057106882327+0 $.1630713305653662725772673+0$ 1947610974432407442518830 $.2120368987604358993354671+0$ $2198079554326709593010943+0$ 25054651 $2232965301127648269570040+0$

$N=12$

$939475546882713185755319 e-2$ $.4885804340937446348471456-1$ $1172862757957247918016400+0$ .2104545570059180564392585\% $3225648853337444699080334+0$ .4465428866931218281307671+D .57441201181072120770660840 $6977755779044984314317850 \%$ .003877516320434331380999\%0 $8987540653327876978445911+0$ $9626935959615715229970911+0$ .9958064549069209421871208+0 .537507202829e320269517046-2 $2810915250282973457079276-1$ $6815424584800657337585390-1$ 1240874584649518749900241 + $1939096213440696664295713 * 0$ $2751170979308180735066893+0$ .3647760005920754806143029040 4596146246281561328730855 +0 .55613076407532476499336ce+0 $6507180074834021521628846+0$ $.7398045472525497040930133+0$ $.8199967771198717626798682+0$ $.8882189486991781674602966+0$ 9418410249417674523104717 978788298870064904739552040 .997627767338738742467623240

$N=2$

$2429978828475440309454799-2$ $.1275612399629951258068546-1$ $.3115782404655719067824890-$ $.5733823454055490258513748-1$ $.9087096194840292993240761-1$ .1312095640R29700R98396743940 $.1776963196699771916489667+0$ .22957206464873914320275700 $2859872504614115078029894+0$ $.3460143318228104664549461+0$ 4006615998702031901413841 to $472888506995724090515173+0$ $5376223899143700972199642+1$ $6017763464176344056467814+0$ $.6642678888267722075283289+0$ $.7240379323996112469860662+$ $.78006964764068439221000342+$ .8314067247073703 CRDe28 85294 $8771706348357654398170683+0$ $9165765211377072282786839+0$ 9489474005895053985918391+0 $9737264074306179008332503+0$ 990486854799e279873177024+10 $.9989399366660609810408788+0$
$.1376598464740006350000054-1$ $.3157508628720544671779414-1$ $48224389 e 6164452030717801-8$ .6331 5924199500430991 seee6 $7624917075842071955156497-1$ 8606953556027315479151271-1 $9515922837661942764637529-8$ (10031837050513901615083040 103183

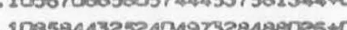

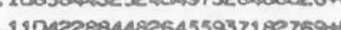
1104208448e645593718e7690 .1135051408012146159691636+0 $1120091891610919185913052 \div 0$ 11237 ree475631 CesO42797172*0 $1124858459068593553511341+0$ 1125227493785328006014949

6229331762389439765950189-2 $1440195242418184215649020-1$ 2235243924450980659819572-1 .2994073872269130412501344-1 .37050 C2319528001995085645$.4358034988835116161005899-1$ .4945463813378257571274455-1 $5462546105402723985799879-1$ 5907656104242609514024046-1 (28000999896850797110475-1 (1) 6505 70 7 7180619634586139568817985-1 7291478556087100795467687-1 $7371779959267327551023791-1$ $.7428060234158851816875209-1$ $.7466004105766723379620775-1$ $.7490408914754268788818152-1$ $.7505209773353856705695703-1$ $.751353752030020490617649-1$ $7517780655404729064110906-1$ $7519693055044354826765452-1$ 7520370465441798528666037 7-1 
$1245179134435040244396394+0$ .729083 roe $1604849105558436+0$

. 56714464065386053947195940 3845947991298865C2757815540

$N=4$

$6620016830784749389890308-1$ .3137372653784858805014977+0 637333186239062185774548540 9000244738525026819403301 t0

1656934398410866831255331 to 30421337758640106866474494 $2706455105812883441206441+$ 1007569351864964607940994

$N=6$

$3265016120507337780840628-1$ $1636297885906563589890199+0$ $3671361940733304348636674+0$ $3973299786630457034259124+0$ . 8052299694asce $14662385373+0$ $9488328821876800658801781+0$

$8281429551661712180297748-1$ 173606873640033635413559940 $2195129152302947223176756+0$ $2012759089066132717405893+$ 1256668806154776117658401 + $.3843238928623619366436911-1$

$N=8$

1934656167936207757272972-1 (50) 990187zsezses172116918954-1 2scedrs $3969 e 20717426023399055997$ + .5752674877528983025006790 . $.7431621970600495053513068+$ . 8799190591321317956729686+0 $9690652760533958250658543+0$

$4931331824303393679841235-1$ $1081739676403133092758345+1$ $1514504502617981717881725+1$ 17044560123996665703535904 $1591003848655220277664058+\square$ $11988670609 e 0149889416669+0$ .6506658850952775093988879 $1837224634309571415927157-1$

9056647579583088582247288-2 $4708750867032975062168546-1$ $11296864289560 e 2166100345+0$ 2Ce $5246303277493996637521+0$ $3100800545698904280931601+0$ 4289e35681712044714730553+ $5517319234523368407303446+1$ $6711040673758606280142086+$ $.7799612460795478509942482+$ 8719333282123326248981670 $9416890927288078833690682+$ 985210472508019445677902340
$2316990845081379098999374-1$ $5250538335013947873397337-1$ $7849546315493191270086718-1$ $9919309759061723038438132-1$ $1126672241820038586382610+0$ $.1171490517914960002416213+$ $.1115679434129505966942145+$ $.9626752957735582105026241-1$ $.7350748512900176678957520-1$ $4740671496296363118275478-1$ $2322466711095466042813672-1$ $615479448004380987096930-2$
$5227959780530207454999262-2$ $2733674290961945400013557-1$ $66266142453315922519 e 2190-1$ 1206046761762855407015243 +0 $.1883705498556211470321585+0$ $.2670982014513314959182705+0$ 3539414243416655439082627 t0 .4457901255296638736811900 $.5393912277383994409714323 * 0$ 63146650717562311425103694 7180237854320323059501037 790460193287075151575863040 (96765672 3501517430 se4062903205579e32791794540 $9657690685874632080037 \mathrm{CZO}$ $.9913698122829605273071696+0$

$13392715836907515034009 e 3-1$ 3070ezz991546a51960a5t2B1 46912621 1 599sersuse 4525275 .61372038121698 70621835229-1 r344644623057134954268726-1 $.8248049 e C 2595251187661479-1$ 8786501279a12335100577276-1 $8904561872582474309630573-1$ $8574123265102774163103146-1$ $7805283806633371764297321-1$ 66579157800111305900031 (1) 52439036r740752421z08etas-1 17. 2265907115084945477547454-1 $1066029973847135256221360-1$ .27575282428262135525326557-2

\section{$N=24$}

$2384317521299020051288686-2$ $1251962720725596642954834-1$ 3057820623969246018614188-1 5626590911546632136424419-1 . 1512 128712969457059565104564 1742749124369632060974863 + .22509e7045659366332346940+ 28073299e89816693446678810 3390808126799902552188817+0 $40038637272013384011501618+0$ 46325102371686985910395990 $5266590377341232458088721+0$ 58959190937953558179166790 65109265731663849473359784 65104265731653 1.

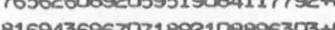
(169436st . 8631760606964236057497395 $9035955475035686094451520+0$ 9375671235654964891378251 to .9645577917305635933491144+ $9841445229716109399633630+0$ $9960205610306135219204399+0$
$6113993099244821960953168-2$ 1413431694865500947045019 2193310987954520235905009 2968595373002421839877 3631070 4250110 126301001 48194654116ee937572833047-1 $5280913461665508035494163-1$ $565169559834750141296166-1$ guece23810e26121171817134-1 $6007737654356975829878755-1$ $585309 e 795436701176791383-1$ 5560144386174354917542182 5137839070053250979793 3979177009007 32979 3. 2595007140575991551149700-1 19001975739149705-1 $1281554778112044601631509-1$ $7479294539053714968993715-2$ $3412718594797478110511767-$ $.8667444736337065125761007-3$ $5900667658236846051012453-1$ 460971000057 
$T(t)$

$N=2$

-2316301 98305085193428065840 $.0889762574539 e 22138950 e 36+0$

.5516595390407090401 46388540 $.7996691181052700650860313 \times 0$

$N=4$

. 7277400055007901 62 $1522900-1$

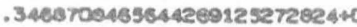
- 708114480120494013278365 . 96334341339230054107409770

.182388179611782583735340040 .3488285057505288090205182 to .393241691084872875455017940 .367870600658375557001 7437 *0

$N=6$

$3485173125557142731281620-1$ 1749882386315915833631 se940 $3898074083530738367084769+0$ . 641737987624993399822850640 . 8575634164124579e80872168+0 $.9633630566221013906068488+C$

$N=8$

3843013075755013729046915-1 180810938028548508313320040 $2459021053337356215535578+0$ 2659393752418354633443651 to .2645479871101195446906652+0 .259578242674270630040140540

$.5104036125414334056201225-1$ $.1139587670679674370472625+1$ $.1139587670679674370472625+4$ $.1613009010587979044010186+0$
$.1893547135053954589285090+0$ $.1893547135053954589285090+0$ .2005061507333642056987566 tC $.2016315482594480993491039+0$ $.1950449306108860592397948+0$

$N=12$

.2396269127238288501275236-1 $.5434176851803615658554036-1$ .8138173830717595434183212-1 103453204525806142246891040 $.1195493063158405266423300+0$ .129572e2726671372909621648+0 $.1343949610105444212658845+0$ $.1355190713812591665300667 * 0$ $.1345406462694183752769065+0$ $.1328069300951851116 \mathrm{cos} 61$ to 13120569915009755780967

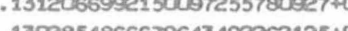

.

$.9957706104214381936614374+0$
$T(I)$

$N=16$
$5363721599362791640284820-2$ 2804e96se $4445796310399035-1$ $6800414276592082798779930-2$ $1238024685433812891081835+10$ .193443331952807872562825040 .2744319170168170091744477 t0 $.3638627314090510373065440 \%$ .45850728725002456158068040 .5549113307976360899691061 to 649490943909194555060902 733705543190574939550090 (3a)

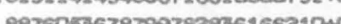

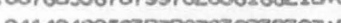
94149405s63070870sezsesstu 978655295933621975309058900 $9976125006234840498250506+0$

$N=24$

$2425835359089000382910457-2$ $1273793657151040334049450-1$ $3111288467700202621255110-1$ $5725401491664381527615139-2$ $9073412254998132762967574-1$ $1310059949637677896090252+0$ 177411852201126005311385540 .2291942266157962604909415+0 .2855077 134260038168776594\%0 345431636209014170642078040 $.4079833019533205772171244+10$ 4721320155715695396557396140 $5368142406568784699592195+10$ D0099594325413610706614240 663459066159406319426116440 723282536224055850016538040 779398615500614442975563040 8308432729883367373655738+0 $8767280636397445933818945+0$ 916257130797198845099096+0 948742486629798872503843040 $9736172284448392699205196+0$ $99044641261 \mathrm{ce} 434692363390+0$ $9909353790030457575600607+0$
$.2374075820804077635997447-1$ .31 909501 es .48165554396449472042387r9-1 .631043139980640 es rezes sez-1 $.750 e 0723875 e 7391$ roeez1 563-1 .859679916527057 4321209732-1 .954206100951830971 5690468 - 1 .98011091287rossecoertose44-1

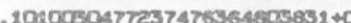

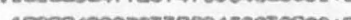

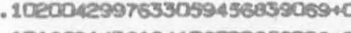
. $101859143819410802355025+0$ . 1010620303695790560053035 + . $1000121020570904535952462+a$ . 9900245817370008274650027 - 1 .9623110065381250431 7es479-8 .9781757r775200s41 seces4673-1

. 6220490356454103550504615-2 .113822495250 Co1 5834845755-1

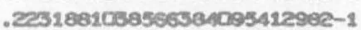
.209sertr $40423755431295501-1$ .36977990662 4999azoneseses-1 $.43463563519 e 4814653463833-1$ $.49 e 525072741987$ 7 $968553 e z 37-1$ $.5427045317787278124963884-1$ $.58472399945731150950300000-1$ $.618479312 z a z 8014251605964-1$ $.6442396918774921015276560-1$ $6626187066854466123900903-1$ $6745069041793540000313560-1$ $6009709595999031039006665-1$ (1) .68s1471121 sel 6745554as 67455548135914se 58937 7ro63-1 $.6055050354448859407785153-1$ .6646005534153252 Tat928578-1 .601e3948839e7204500440237-1 $.6566559244425246597530933-1$ $6541444586926673242177174-1$ $.6528530866620744728659747-1$ 
.197423794518750596215972940 $7363200025489955514029448+0$

4655093301964751153818097 t0 .4085098545675648214398035 +0

$N=4$

.6871 8439C2813787620345253-1 $316490 \mathrm{r} 38500143725160001$ t0 6414702567330448965993946+0 .901 850e275330637907392523+0

$3282449145482232564997399-1$ 1645854675152141536378311+0 $.3693795247848960463213310 * 0$ $6002817721648648647435764 \div 0$ . 0074115889905357861359224 to $9495446108446129332923910+0$

$1942520141529435294409544-1$ 99443 eee $51406683185089363-1$ $2319197278994014769457291+0$ $3987718486898717747730782+0$ $.5775420360065801577480006+1$ $.7451580368255965248072726+0$ 8210939400075294263991941 +0 $9694085376125641692347153+0$

$N=12$

$9081625243256722896068787-2$ $4722051495678480760727549-1$ $.1133032347146602956248536+0$ $2001593910790823337759724+0$ $3110893866019913446425329+0$ $4302725424106819869554738+$ .55328n875999411366428175940 6726227736886722132128896+0 $7812206713912676424811427 \div$ 872787666475724377589091440 985326338176256116891987019

167072828527070358638014040 .31016492592031363363437094 28596748 2859e74812041764659755736+0

.8926705559622805018908282-1 17504177750796809417732294 2242874446510954033681721 +0 2116428118176476084722913 $1368097432847228295498204+0$ 4297035190637795106402362-1

$.4951624946233404137005629-1$ $1087190987556033325146374+0$ 15294131323098001576700594 174482006475521239889687440 $.1667782489885567568343932+$ 1287989459709585213439622 + $7206635345043414406078184-1$ 2071688242971188504108901-1

.2323413985228197981026063-1 $5266109949015637064046647-1$ $7879716147785233806827536-1$ $9986685735828600275083932-1$ $1142237104171352888480175+0$ 120R $4144027003369646769067+$ $1165272169528736613666480+0$ 102641815721896764055538640 $8004726801320293952637477-1$ $5261557786525529613676088-1$ $2616351966249146671631370-1$ 6999377682250864225211225-2
$52388800031916714983811427-2$ $2739459335892662149545982-1$ $6641016141608248718859137-1$ 1208773707265071 55444860940 $1888155723592493269615684+0$ 2677503490542639184384173 35481Cе6епт7738912678516740 4463458535379033140541219 .5405624097201343646571991

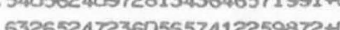
1961 119910914626777615926602910 $7993095451731488 c 53810008$.

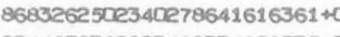
se4tecs3eese3 489334656035 t $9659695553623580566310523 \div 0$ 9914218919211441955500528

$23387672779195479726520033-2$ $1253733915569277575134300-1$ $3062196072467974906062373-1$ $5634786189997509967073675-1$ . $8929172931319096671696595-1$ $.1289105405841534559330915+0$ $.1745506061260063189584691+0$ $2254584382056001839489014+\square$ $2807937060964253575960066+0$ $339644219 e 01820477142074040$ $4010426143953767085647880+10$ 463984156217783439855237940 52744505439605545751563 $59009576906866311906681+0$ . $6518384787695364557562333+0$ 7107833958612919628206611 to $7663021894154921377519060+0$ $.8175222197319197284964807+0$ .8636426123138325744672855+1 $9039453087652278701321110+0$ $9378050723789461462899838+0$ $9646980190430999535300406+0$ $98420892826188375703334485+0$ $9960369772784407389501057+10$ w (1)

$1342077002401426204394644-1$ 3076063205849485166371 821 $4702583283654015192377642-1$ $.6157021407626646221132632-1$ 738306330376613 3624417644$8328851286835142775829236-1$ 8937256235627a62558503759-1 9154912596510011397705300-1 (215491259651001139778530a-1 $8937060074492539138415139-1$ $8265610463633997414370900-1$ $7169556718206446708912261-1$ $5740001361970563610952680-1$ $4132146848204729345341804-1$ 2548793510227340326662459-1 $1210334840969214829096594-1$ 3148882509284341675161069-2

$.6122807310795912904555501-2$ $1415447715261713974120604-1$ 2196541808346776646552949-1 $29415505037045358395872903-1$ $3638023754219567117567382-1$ .4274119693369322756040533-1 483e476050695132559350565-1 53199e61se693544625110906-1 .5707187329531747679969179-1 $5980690618527008043672351-1$ $6152804689498367183115263-1$ $6180654673820973947939941-1$

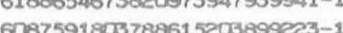

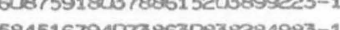
. 54632862553908त05e $1611835-1$ $4952094764797752315424432-1$ $4331189815247013574343679-1$ $3629792369879171083324069-1$ $2885719917938712258731853-1$ .21431639e51357838684405e8-1 .1449463859781906854130704-1 851218270ese24765871410317-2 $390173285364161875723819 e-2$ $9936824534990447383697103-3$ 
(1)

W'I

$T(1)$

$N=16$

(18) $1851978002913559900953312+0$ .2413127540563494242581581 t0 $.2504770241229544842489740+0$ .2327459354897027858484999+0 35440979e 4098136674234587 t0 $.2167036877233290558350055+0$

$N=8$

20241 42500063658062619342-1 10365661858633879930930464 1036565105005307950030464 $2419436207017047237525148+0$ $4166573401290029870433783+0$ $.6045203635673331582034694+0$ $.7796628154694230593708342+0$ $.9157667296761967301394336+0$ $9303514267781723841627602+0$

$5160038594556015328914058-1$ 1133847276574747003465534 t0 $1600835945713090655364412+0$ $1859291321566303106017261+0$ $1906568817477191072031545+0$ 181563911278520 eger2 48961 to $1693780894239286477538975+0$ $.1617286011626485289541615+\square$

$N=12$

$9339328521134557282451370-2$ $4856504098397518720138830-1$ 11655e8 37833381415563950 209053128713362919046969040 (3) . $4434076804369578485004445+$ .5706512113330910806379781 to $6939798832691580721760148+0$ $8052557693724250451817117+0$ 8967627815081241706578995 + $9618689889121458455218392+0$ $9957083901729508527991001+0$

$.2389393891292663344950964-1$ .5416747728559574390458 .810956779950430e844644799-1 1 (1095) 1950 126970 12657018778se378988511544to . $1288947829218173402447284+0$ $.1258824054659614489728578+0$ $.1203155242449796183664725+0$ $.1144482159786554418766757+0$ $.1098515170235062445182999+0$ $.1073801902553006552307126+0$

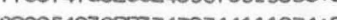
(15)
$5351113009427072608442839-2$ $2798258465146087668230399-1$ $.6724091218342635634521003-1$ $.1234979286140805703158452+0$ .1929491916472123105349557 0 .2736960962637372392879847 t0 .362839829757630089777914840 $4571847529093397375210405+0$ $5533389140000098023 e 641190$ 553338914000009 $6478052277124858827437712+0$ $7370731057314719776452986+0$ 817730197621549201057267040 . $8066027466593136263792395+0$ $9409124766791088735150425+0$ .978427296815460424762686010 $9975861131075394273035640+0$

$137084902520893609173152-1$

$.3143065893946315022316427-1$ .48046381 4452359e995317756-1 .629323895811 7820393657079-1 $.7555418103775377890406364-1$ $.85474646222330230230948059-1$ $.9240197342630746434402978-1$ $9626708794030849642406376-1$ $9730415879197964684931300-1$ (c00618094043370154103532-1 933254071119060506974023-1 (5) (5) . .8148061961589674737035017-1 $.8040274778239699600672771-1$

$N=24$

24220008286060772050054837-2 $1271775477525497549545132-1$ 31063310390006347014235 $5716190553300053670593080-1$

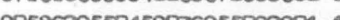
130

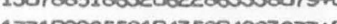
1771099655810435804963677 t .22879er2 192647805848503800 +0 $2849099071662064658939877+0$ $3447847755082304653840692+\square$ 407200444663540484428234040 $4712171585332585916564732+0$ $5357860526301514757894338+0$ 599842990029004441323352540 662322601578724802054051940

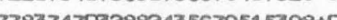
(2) $9157285004212557257250000+0$ $9483976360616956069622984+0$ 973431257374147912849539040 $9903769817821039656283918+0$ 998927524132562488103536640 $143583790487939090505351-1$

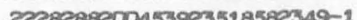
29030

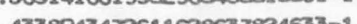
. . 5 . 5817 re $4568134060157939501-1$ .61323629966072076338960e2-1 63534Ces8348540e234838702-1 $.6482516855195177557517142-1$ $.6526747148750469364294950-1$ 6497665266312900506911039-1 641037797672150981000000-1 (5) $6129118953724405826466743-1$ 5967582627610940706470057-1 .5810186850653308137829e0e-1 $.5667018278654232346787269-1$ $.5545466539230632231591124-1$ .5450634773204470107835428-1 $5385825842902753968113112-1$ $.5352977600294919347137555-1$
.6210656995012059540275417-2 109 
TABLE 6.- GAUSSIAN QUADRATURE RULE FOR $W(T)=\sqrt{1-T^{6}}$

(1)

$N=2$

200589786916898343240011340 $.7455726600730123736010423+0$

474099180653025334304027040 .43684480430481 $77701284512+0$

$N=4$

37ereosores $72392283597651-1$ $3195667357275720163337810+0$ 6A7200337375250130948e56540 $.9047467242100877677667062+0$

1685447083214997227959661 to 315040413001693366579710640 30e21748e39003069664484664+0 .124941389244343049608334940

$N=6$

3301885843412491154815001-1 $16561485967880180796 e 6461$ t0 .3719440513073763263104491 to conassazsea r963247364453840 8107503487088211779818626+0 $9507043728538749152835526+0$

$8376507044377595806290145-1$ $1762928849233464878475253+0$ 227878321326585458549552940 20189578ese687405069740 $1511573069865178104100606+0$ $.4975462659074335969889559-1$

$N=8$

$1951306825743082864735983-1$ $9990659271881994909911413-1$ .2330751160369460966485501 + 4000198967214111018079766 \% $5805491864258133907482330+0$ 7481094100782634165877557 + 8829642543544963105782317+0 $9699753665725955758262449+0$

$.4974148298505323155883309-1$ 1092515600009633718450118 $1540142521790 \mathrm{cec} 907612533$ 1774373905540599103919 17413275sess 175 s $14005426 e 3535004265461363+0$ 2434174219751020619756674-1

$N=12$

$9109558248461558923743778-2$ $4736745351176233563132006-1$ $1136651007748527051514341+1$ . $2038391400794882179634748+0$ 312199075077751084247818500 43186845389581506549767390 $55528 \mathrm{9} 3684426207181720424$ 6747709622272935615857870 $.7851300393106780384406737+0$ 87414837517654319519876784 $9428281688576118996431721+0$ 98552012200485731678428064

$5996132606338093110073569-1$

$8346835633964064205591725-2$
$T(1)$

$N=16$

$.5251091930692792494516502-2$ .2745806091369993856941 566-1 65568CR221 4227931184171355-1 121171353850120405009960940 1892912399212662306598531 t 2634561 1899470ee689525087 .3557879571662728275457087t0 $4481079472745539635190390 \%$ . 542064744096787372963136040 6342828457076905365824792 미 7215137129715631261045821 to 8007647651971971503361267 40 869392636580536363474401740 95164051214914190909425 96saris 991509527090153535409554240

$1345209969399729269995129-1$ उ0A41 42723 257265137219400e-1 $4714002576020560967595744-1$ $6173490595379547490300576-1$ 71050 137450

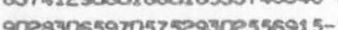

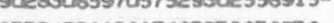
$9332402812810482759038738-1$ 9244881495730857576422790-1 872604768e noeseges0931296-1 $.7758356627515352395935734-1$ $6379009737986536878468316-1$ $4713816666960182250579699-1$ .2974742535663579066706409-1 14377 70sosso4ascoeer13326-1 379e410391052051299462033-2

$N=24$

$2391423526684103488320166-2$ $1255700973557172596019554-1$ .3067046207907868337713067-1 .5643787845168766420827134-1 $8943619453818405740777429-1$ 1291270593076327659571564

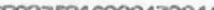
17. 2129090795000071131444 340271sed1389675172097809n 40180136353108967836668 52844161876074391876057984 5914748548838299611226788+0 $652947648180180 \mathrm{C} 211372656+0$ 7118739053161200337489638 76732040165710075944617640 1070 1 oc438s

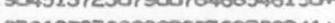
938197936e0sezrnosesse 41 9645322830534519618639740.0 9843173853240056552901910 $9960647578184413762566587+$
$6132231864223953989692880-2$ $1417683538270003940569387-1$ 2e00035167170374220707319-1 2946352085805960501065262-1 36442426919e1 $100927537350-1$ 4Z8е18creases $48499903940514627469519-1$

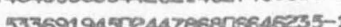
$5735469510009558509054-1$

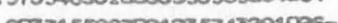
6007155087 Le1255743291026-1 (3)

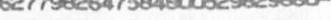

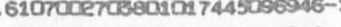
$5797403589663950242497006-1$ $53475809 e 6268904485392416-1$ $4764997009637507205974737-1$ 4069459030129173643067890 32948479549e9462395818544-1

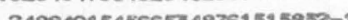
17090150513 170 165 $1201958853849850877296654-2$ (1727265679395716748727-1 
.2253960671 8959225354965231 t0 849916183532756990633479640

533916778688114965965549740 $6296757925301544093369684+0$

$N=4$

$7176295263900086991529586-1$ $3413323115899666792237316+0$ 695236239930197121 $7720230 \% 0$ $.9804691020524162648190490+0$

$.1797881131303277705361749+0$ 33778409782399450651551744 $.3512912563439316855275457+0$ .2947291039200154127032801 t

$N=6$

$.3452139477390534675785993-1$ $.1732177736598475398629786+0$ .3894747177630029138281097*0 $6345412420716818955136444+0$ 8519e949es35914610661633+0 $9 e 24606676647490474211754+0$

$8758359092491355523246647-1$ $1844983465201171375086967+0$ .2397494184861640564114986+10 $2442527628940114022470505+0$ $2159892307850513821211812+0$ $191519 e 216080118417816248+0$

$N=8$

2018338682681CR6585586613-1 $1033803072806932965040906+0$ 241269252265340032449533040 $4153774454938662682753184 * 0$ 6025033981157713805308142+0 $7774563396516134566453904+0$ $9144718723516904413655563+0$ $9901704416964351778327957+0$

$.5146468835733052233122436-1$ 1130749545770275298893705 1595763126853763015016761 159576312685763015016761 +

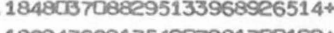
$1809476691754207981700122+$ 1721564072368918204384287 + $.1535395861210096977005078+0$

$N=12$

$9322753745726375675824757-2$ 48478239621508044599e4433-1 11634139736090025481794 116541397509052 200673158595789310008376 $31969318 x 2596044761057854$ 44247 rescar 47916266180062 569371681257872159898345040 .6924735180409074240565064 4 8038303663383317194531057 t 89576093975378497544970e6 961427290252666369213061940 $9956542896153438813700887+0$
.2385146651163786116292117-1 $.5406976019336749636371705-1$ 80044248747 $1181341055436329574150682+$ $1262399319742800631109162+$ 12703438590613499380116294 $.1218096774116101643310283+0$ .1132185990CR667554341108040 9744006342540497916343516-1 $9377543271589204290841865-1$ $8094424874703764950963775-1$ (1) . 104326261224730603758297940
.5343932CRe9438403261691341-2 $2794488267246946947829 e 90-1$ $.6774881286330022940703297-1$ . $1233281490855831276523778+0$ $1926784818404740875679212+0$ $2732993745305595406772107+0$ 36228803747701 78991 594542+0 .456448391 12279211 86208830*0 $.5524064980990168417994245+0$ $6467143473651279714280540+0$ $7359 e 92685280644784435120+0$ $8166860050514728862961848+0$ $8858010801406381776639632+0$ . $9404271215434436894337888+0$

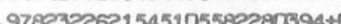
975632481364243534900024 $N=24$

$.2419817710567806845254686-2$ $1270625882424325456557334-1$ $3103514199177617681273783-1$ $57109878826201680211601333-1$ S050318203153569674979254-1 $1306673694391977062350966+0$ $.1769437322109700522788310+0$ $2285735324844436076723154+0$ 284710 UतC $420195400653695+0$ .344436087330ens221 59191 Ce+10 $.4067729634038955206033572+0$ $.4707033204415411610017633+0$ $5351836501402536091894047+0$ $5991598728838550647189528+0$ 66158CR 147611035757757371 +0 $.7214070972128249941041801+0$ $7776296296442802816990275+0$ 829e78548641316154634653640 8754443146924332013451420 91529753189371199605984744 $9481098532133713975802415+$ $.9732734250847837980318468+0$ .990317411264442888112723740 9989207487226400543359058 +0
$.23689996009953713894610340-1$ $3138340048071285206430314-1$ $4790095344796925397500563-1$

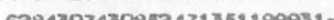
.7543935210006 7095064695 $754393621004650 s e 86469272-1$ $8531818727487739050700088-1$ 9213948788893 $762427280034-1$ $.9572101837156037163311901-$ $.9612713549713257604248359-1$ 937772372481278061 3322739$8947141936531675007382724-1$ $8423872675160439632233471-1$ $7907048907206236539766 \mathrm{er3}-1$ $7471515900031450010050000-1$ 71697 16e974

$=24$

$6205050375806400167151140-2$ $1434537145726216283879411-1$ .2226256397969507127467375-1 $2981555613827484820009854-1$ $3687961554553926778181727-1$ $433387930000568130161740-1$ 49097806373541506330е864-1

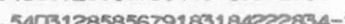
5800105050 610 6120097945115497146569145-1 63res6774736rscer4867249-1 $6445830812751339387675432-1$ $6462199433108763119791951-1$ .690571116623033234126172-1 $6245144426564912471095966-1$ $6044971076956346216627875-1$ .581179653e19ee?873509034-1 $.5567258591720055550211006-1$ 533070 $5116363575850905163090435-1$

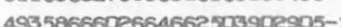
$6133997612562349-1$ $4652908858721676319265416-1$ 
$T(1)$

$.2023750635362043005080766+0$ $.7520591823118005787124972+0$

$N=4$

$.6762020686854779647513202-1$ 321253416386969380133200040 650931087602340515521835540 .8070043041 79646zOs266680940

$N=6$

$3313027475668341321183687-1$ 1681895318552647647412909+0 $.3733520723141671633334912+0$ $.6087400623248180999823801+0$ - 813e2 1290859es817731 50881 to .9516393975671376047068676+0

$N=8$

1956344262078435102748300-1 $1001683179778967273816619+0$ $2337093202421058867791785+0$ $4021048109961527789666053+0$ . 58239e6129399936559551820+0. $.7501944930315226764644000+0$ 884422559463840088e269185+0 $9704398384684984369409855+$

9125565122275229627343619-2 47412165447 $113068786661125451722562+$ 2042136779918064104489568 $3127954785581012200101503+$ .4327561644352037276574994+ .55649e9er9573438245021162+ $.6761952480091170977483304+$ $.7845189000322064234013185+$ $8752077517796296135575088+$ $9434040098971910195901522+0$ $9856813991375511742739003+\square$
W(I)

(I)

$N=16$ 6533254032171594767530392 ta $1769342865841215075658857+0$ 22917732252233383328082682+ $2262440790501443410582448+$ 1597483Ce 46CRE364746662884 5472082444512613344291946-1

.4987c0912399348e666537866-1 $.1095438518452178859518468+0$ $1544817045074620830549787+0$ $1783663980062306759258848+0$ $1768156337802332964489838+1$ $1461019285986481288694083+\square$ 8858634114313400008030479 $2710790785376460524522864-1$

.2334679306084943762837985-1 529e189356715093855200440-1 5ese rse135se436e64276396068\%0-1 $1005217345566718070102887+0$ $1154864769856860636827039+0$ $1230779583387508235723436+0$ . $1224393795865288369901904+0$ .1126179475C25951044298217+0 Se95187663587300418967146-1 $6488855002336285321680844-1$ $3399186635382039889602390-1$ 9415987925061955675749333-2 $1292417016676006735154758+0$
.25800686423312055920115327495570163505634909e7281$6665763971437459200311490-1$ 12133638310114253839091804 1895539167678606151586221 to 2688399770836495414400037 +0 $3563196451289120420021755+0$ 448814009512414074937111340 54255450 5 rez16473245ose 1281409782 8017948431570569432517410 \% $870221915612366288 c 83397+0$ Se57169023530737913066463+0 .9665772142195598758646960 .

$.1347003381579255401527408-1$ .3008284802541602794364937-1 . 4720509826544924450457275-1 $.6182151044238530190603770-1$ $.7420031616977247969683565-1$ 8380976842625197297529103-1 9052494278525999720970196-1 93a1150051972 13513-1 3346650447570131313307361-1 291565659294131 $1824227053-1$

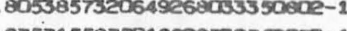
$675715587501662973272073-1$ .5102490594583470374182135-1 328660966027307779570R 424-1 16149686721 76494128266399-1 $.4294096317040820380014180-2$

$N=24$

$2393570728835934495465314-2$ $12568382666854397801943109-1$ $3069812789406790296946786-1$ 5648902164668579476112148-1 $8951777034604105274728961-1$ $1750079172634822436804181+0$ .260639583677459347171650+ $2815699755360449733519266+0$ 3405122049456964283938684+ 4ne22176008435241666455611+0 $4653696792025484559462736+0$ 5290259041485420875628999 59213700049276561739526664 $6536681306503590327856844+0$ $7120<05$ 7680523014950836521114354 40 $819094003383210571707650+0$ $8649624961387718617281988+0$ 9049677505743729235272227 to $9385186698060530350547735+$ $9651266397406279838707362+0$ $9844083668641725384371393+0$ $9960882103028663319616230+$ 6137739908750e04505esos39-2 $.1418963084148020463342254-1$ .220805008572059e078733463-1 .29490601206326731 $1883588200-1$ $.3647629974569800405851335-1$ . $4854422827242185549 e 32512-1$ .5342745042256629671156613-1 $.5743072940241682907496560-1$ $.6048467770287235681060999-1$ $.6252950454379717638162151-1$ $.6350917747744361379165615-1$ $.6336280525153360015031290-1$ $593809013913053305715067-1$ 55403770907 $.5003878750630705283204224-1$ $.4337856431691832145559328-1$ $.3566890077899422388875182-1$ $.2734596744591389112474242-1$ .1962394494156246980952381-1 .1143554180e23441764093889-1 $.5333525376598788243009912-2$ $.1372946902961105627644295-2$ $.4286256673848763307263197-1$ (201725056325897133003173-1 
.2305021220954472145806203 to . 035294072161385473086725940

.139e129e33523042851 524713+1 $.2036521364364538760295228+1$ $N=4$

.3587125582932102832876868-1 . 2939560710570540734322316+0 .876301780850084260481157440 $.9503732452797812837682852+0$

$N=6$

$.7471880363857365358553503+0$ .658579394674575050833328040 .5451246934004284188909391 +0 .477658523426843606270323940

1830137488570383040e37659-1 $.1407897128148294074366960+0$

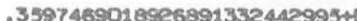
- 319507797051923139630744040 $.9821938708504399052016066+1$

. 5080762233194747442478314240 . $4777325646804528407385396+0$ $.4777325646804528407385396+0$ .372535143528275770732987040 .335e997355697048e49407952+0 $.3117252206724804584696497+0$

$N=8$

92919418265619033199e3069-2 8166048171091533857020068-1 2162471 4a773ees3373723057 *0 3940389663179070902833090 599126878479e185760502740 77705851405 7r17856551 44650758362453640 P130s0515431685046958087440

.3e4431631029s9e8436073746+口 $.3709115736153533562649943+0$ $.3458423218503769927317627+10$ .3143703092123741543738379

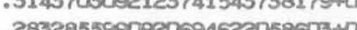
$.2577958655217896138176260+0$ $.2403670962524762517160444+0$ $N=12$

$4182797654613511262828163-2$ .3723863509774276958814549-1 $1012156291669530271748544+0$ 1919898451304628650608087 * .303687657815539e305757587+0 408991762172901421456890740

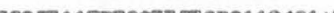
.

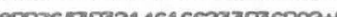

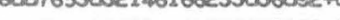
894565727353770751596295900 S611110218468115377298035 40 $9936263595197316077650052+0$
.258347163200365857331433040 $.2541602290311987910205067+0$ $.24598379288299936620355372+0$ .2344101057468472728018143+0 2058978998127005006687937+0 . - $1916574391246276673771197+0$ .17900070a7931ce4703817512+0 . 160950e $04813483415365915+0$ $.1557550063409237765498344+0$
$.1531671929634917653944373+0$ .2205448139e0319987579001240 .1687569506336595171441316+口
$.2367407232573675923254878-2$ .2117526343205507201766248-1 $.3809629413004753512523175-1$ .11176506212500046252547490 $.180215648 C 345581976511365+0$ .260907934467450e1337067323*0 . 350844371404994529652187840 . $4466481420507367879554784+0$ . $5446767506204551688124804+0$ .641157641970eS $0967533213+0$ $.7323364610513124964112125+0$ .81 $4632370083199985595794+0$ .8247899667635598184153690.0 940019015600205771190004940 $978114505050953 e 59879450$ .997551909674271744199569240

$.1058551256599176188873521-2$ . $9500470636577049436043733-2$ $.2624356686323587846405858-1$ $.5100890468044952129995145-1$ $.8338025637413211552596110-1$ 120831002435605419275091540 .2802055297233382013040597440 $2765085968459051417058345+0$ 336657847029490052855369440 .3996397420279969661928756+0 $.4643872636314025802534917+0$ . $5297973079612462304461522+0$ $.5947493715454998136829500+0$ $.65812511679908899753950110+0$ .718828367466935257718521940 $.7758050151206948194015029+0$ $.8280623494487953941100798+0$ . .

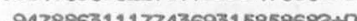
.97317539ce181926832464217+0 .9902861 523274999697271967+0 $.9989175058794761117551852+0$
1944735729826003951089245 +0 19967524641495637605119770 se91 (18399rogrter

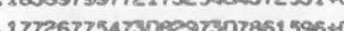
16950451337000

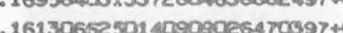

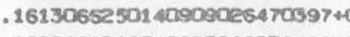
152918628354597090823646940 $144853215550562809625 e 206+0$ .137447407807954792341261740 . 130930192573841480866443840 1254359599956809702382411 to 121ce9655477155934905180040 1217731095133670365800070 11751605135653656000726 11554117a181274854encerer

$=24$

$1300862409144271767790033+0$ $1295333624621600371190563+$ .128471440762427359353294840 $12686097424793300040300050+0$ 1247436651916811822325191 40 12215709017409035953129090 11915752739090837635998040 $1158198118906842047590616 * 0$ $1122335336422364381919616+0$ 108496 7052067136824132217 170 1047082074580395150579562 100960789213165254670 ree $78+$ $9733577988777187218584927-1$ $9390012800373142026738219-1$ .907056289ees32974993311197-1 8778983479768393051706401 $.85178003345876072346931614-1$ $8288568870556051525226063-1$ 8c0enge773725685625399568-1 $70006059801294077035461-1$

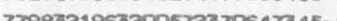
77 cras $7636020069613013542119557-1$ .7603666664391345947791117- 
$T(1)$

$N=2$

2601858165589597964951241 +0 .766536135163793219354050940

243392163737834955837485440 .280206611860463917239621840

$N=4$

$1004363127116205741395353+0$ $3583874894105916415777329+0$ s6secrou4412749030055321340 $.909011699589 e 897449244775+0$

6122403719459e82517444998-1 1775800214978193857435479 2007450562517736848951 840491112633689e531014518-1

$N=6$

$5141004963732342471223538-1$ 1944790437837879315174473+0 .39835517648889100770049e 4 +0 .6208312664270096948201825 t0 O175828834905643355536350\%0 .952239140645367124955228640

.2288126739982375588634277-1 . $7905537440334406253656766-1$ $.1347433688532165788886573+$ $.1488232296794462160470343+0$ - 1041775419179525725270827 \% $.3391799334451566719142244-1$

$N=8$

3106074330719916469661529-1 220е201099563976757871586\% 2559e $19450409879056324633 \div 0$ $4207835167132883042571375+0$ 5942971871195956998863399\%0 $.7556487146290397522942777+0$ $88606894677173482932652222+0$ $9706964079107579419836031+0$

$N=12$

108286600219450R978289193-1 $.3981153478462413748058179-1$ $77019 e 5341757087955076093-1$ $107844525715823915594364+0$ $1170395398796767166769444+\square$ 9749e36845676136352Ce7743-1 $.5691187709248358900955109-1$ . $1671108937365476549656322-1$

$1483351953900109561287533-1$ 5842916906088001 495596842-1

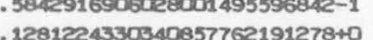
$2196531251903271053302747+0$ 3274580467418496973191181 to $4450681527883464326806727+0$ .565549345871607Z212049566+0 $6819 e 27890961763769920525+0$ $.7875601911036154371419453+0$ $8765153741662579340025302+0$ $9438062409021344479269577+0$ $9857601749552686610875211+0$ $4219546566973848462657083-1$ $.5775937338158370293288829-2$

$639747045000600611396756-1$ $8138436485378838727978176-1$
$T(I)$

$N=16$

$.8650014762260432356705627-2$ .342944809828100048651140e. $7602637632580039082551238-1$ 1323678324333478978290352 เ 2013243878262944039710184 $2804633516807417394500325+0$ .3670118631797044668494902 45796ศ 5500095556705700031493 60593494500741760970893040 15609395010 (1) 60153

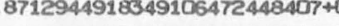
se61926057059761272708501 t0 9667433915380734210999456 to $9916172913719338441067073+0$

$N=24$

.3985644162013197670099419-2 $1587814766243156590533662-1$ .3548517459811710962012526-1 ๓248938454626163380634380-1 $9645340758645334091473429-1$ 1368270142851174719855317+0 . $1829565134066113540478940+0$ .23409624748701 59646926458+0 $2894218732448268393856594+\square$ $348044974920484091910619 e+0$ $4090285083743210917390092+0$ 4714 C65149681493308983371 53110943017140310977473 $54180470171462310877473+$

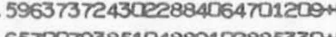
65700073851048691028853304 $.7151369459581137177750942+$ $7698569830619714786784201+0$ .820319908684170089e66598040 $8657455757885147535995462+0$ $9054331910018508022539853+0$ $9387715194045888297214803+$ $9652477945747052088801033+0$ $9844552040694219635341657+0$ $9960988368338968164063064+0$
W I)

$1604245200095555927791143-$ .62755728365515901 13627559-2

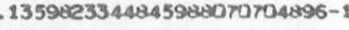
.229019152565734751909851 7-1 .33284213542364351 70037919-1 $4364046182786257153577264-1$ $5272484596391097429372456-1$ $5927169031454303441215707-1$ 59e716903145430541215707-1 $621055601753605563097318-1$ $6077226955619545384669695-1$ $5495057530749846569776028-1$ . $4537375275123545006909055-1$ 334058972367072769e16741 5-1 209345085669e58545e904717-1 $1004581391673182225368274-1$ 2629e21609261959434535907-2

$.5025641124260890880707782-3$ $.1909969036550026241102645-2$ 1909555065580res $1102645-2$ $440198501603539611243784-2$ $7640001897646723429606940-2$ $1156957254471521079684464-1$ $16 c 215369 e 303565014603256-1$ $2079488014255876828804058-1$ $.2565894581451750204921094-1$ .303583320003e429431232119-1 $.3462148006315756214326463-1$ 3817423812216867070991985-1 $4075855923703994545338120-1$ $4215516465965423294615635-1$ 21551 42zor7 4506303555797326069-1 $408454112154422066190039-1$ 38009632 $3411386763245065707276907-1$ 2913940810474475228278918-1 $.235234982 C 066769789219008-1$ .1768329733504397974512528-1 $1207196573267686197496171-1$ $.7138812400880035464828275-2$ 3287908421919193279712396-2 $8397091190358954308721159-3$ 
.669191302831729784946968440

.263110469735180999052921440 .233209015784350029706434040

$N=4$

$7757048244400202192083727-1$ 3 70sees sses85748646153043940 $.743429845 e 23919902674560140$ .962767876322877123974240640

$.1938261129821368814711229+0$ . $4095325338049126187958979+0$ $.4095325338049126187958979+0$ $1393967213871379 e 29883896+1$

$N=6$

$.3633643514556056187995800-1$ .182962493966117461847523940 $.4116135759879848438313137+0$ 685831954681131153724809940 8787962491335016457236070 $.9962865053909609760639616+0$

. $9237938297440646630582391-1$ $.2004257565746102784966377+0$ .290046901064834966851590040 370050 . $3796560304393841575685032+0$ . 5194552906422337267546661 to $N=8$

$.2097341112314532654353128-1$ $.1075970682359741279381245+0$ $.2515179103879648200009170+0$ . $4328372903303850432124372+0$ $.6254016254744798241728531+0$ . $80016092481978710033212378+0$ 930355515758012908571099340

$.5351492433377449548634005-1$ $.1189332968958152935906020+0$ $.1749650449701184611293713+0$ . 2239718463919254186245791 to $.2733854762616458362984013+0$ $.3369878782605101258980994+0$ 45231071170711050003 .9879888455705914683780411 t0

$N=12$

.9562793563395977012124704-2 .4975672391074898176137756-1 .1195115110684994479595657 + $.2145213878199812456646296+0$ $.3287447345064032152720045+C$ $.4547364856764484966413293+0$ . 584119856235672741571274540 81839e340680019930919481900 .907242406055345729e570080+0 . $9683676599428793596909655+0$ $.9980614566948807613540379+0$
$.2447106508548796043843162-1$ $.5563791204311658050665498-1$ $.8415659006599612621617740-1$ $.1095574365039445623726122+0$ . $1323210724965503908712599+0$ .153636972231149515027743940 .175267828680498127847093740 157709 199727cen14ad37614711994t0 $2011649472792023753151938+0$ .2784906481818026698393761 to $.3705040836258699169673861+0$
$.5446744044126310537637219-2$ .2849031076583425111036174-1 $6910080735458836640883377-1$ $1258509260932921175537894+0$ $1967052422607552814561918+0$ $2790820728668401466076612+0$ .369935863917976307186468940 $4658648869844356816889626+0$ 5632397172354233928812175 +0 $.6583455514355559505851456+0$ .7475307573176861755359536 t0 $.8273542397052074850064480 * 0$ $.8947247751180615424736945+0$ $.9477061147477487354099174+0$ $9820216051971771322142767+0$ .99090844757901095588298800

.2450760708120480396760527-2 . $1286983481409620110486497-1$ .31439e1672761707833207207-1 $.5786438410095071697799780-1$ $.9171853884313091320475473-1$ $.1324508995427257308910390+10$ .1793941044465586669425514 t0 $.2317736147198950259094604+0$ .288719349561814364795649e+口 $.3492794587895678132322895+0$ $.4124359994813235141085926+0$ $4771221111344315287763896+0$ $.5422405115915416945827400+0$ $.6066825534135790099074508+0$ .6693477777626274481747269 .72916335264526841759926874 $.7851000795245740287973647+0$ $.8362055939771172653350511+0$ $.8815914207309603435340416+0$ $9204785696745608143815727+0$ 952196382525174768466690940 $.9761973295535414362828306+0$ 9906680966537795088025431 to . $9995144217484676754187294+0$
$N=16$

$.1395462769097641003895515-1$ $3203063269347150330820596-1$ $4914687832290443628018975-1$ $6496250029509099962207875-1$ $7940328575779790047040635-1$ . 100215253513453700050 1169597540353 16097540510010 $1420126694495506979644115+0$ .15711420004716846597936394 1759731908061062845687865 + .2017833074056997940479478+ .241949571819565766697057900 $3211915629010080125732782+0$ 6991409957678901867317743

$n=24$

$3734970665005-2$ $1453335681570205110816307-1$ 225744891480500907848200 . $3029746073751866034422173-1$ $3763272984763575562688154-1$ $.4454569345249206418404039-1$ 51035689353e4862789699352-1 $.5713258561538952119130666-1$ E8ce004410696678381322444-1 $683909505612110930655342-1$ $7372344868471926940617181-1$ $790008287778277429487019-1$ 8435089974817467071151954-1 $8992400515018579791477438-1$ $9590525663253643402706934-1$ . 102529485921057695418001440 110116526481352627952443040 $.1191320405963884890908816+0$ 130309859385604334353362640 1449827003993643973814201 1654763961410642174505124 1979385195350179791405 $197930510055321797914625+0$ $2624375725166264640913185+0$ 
TABLE 12. - GAUSSIAN QUADRATURE RULE FOR $W(T)=1 /\left(1-T^{2}\right)^{2 / 3}$

T(1)

$N=2$

W(I)

(I)

$N=16$

.257007894019030277204321440 . 0135412005836e9075810641940

$N=4$

.7853233ฉ3อ182432946381075-1 365815432775711018196021040 . 340169501750 ๑reseonceer2 $1790078753514+0$

.641984911580965397519369540 $.1461280246407215994243159+1$

$1929856568699660324281308+0$ $.3974989635025475272558533+0$ 5680256510se199899e 560396 to $.9447628865234679328225050+0$

$N=6$

.300e198017706051441781718-1 . 1822590340520309902849000 . . 6601500599582535666353307 40 .

$9155550156709031109046226-1$ 197881251980721919e116496+D . 282513219098696965926855840 . $.4495745095098818365869159+0$

.20E4164908665650465654161-1 . 10688720037 78044626185123540 .249800208652916540825247940 4299305740130407051853090 . - r957cer3459400

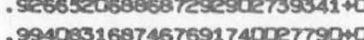

$.5316820947072682296604277-1$ $.1179943583985030242324827+0$ $1726834127852213762564197+0$ $218249093850648958009152+0$ 125070 . $397510161540458030358683+0$ . 3056748839714324063128131 t0 $.3753166298403824954135317+0$

$N=12$

$.9522798806409047438131560-2$ $.4954493127894422114136726-1$ $.1189901697882763172553211+10$ $.2135645322795341638158066+0$ .327262553253767232753725040 .452704887960990976736390940 . 5e1604719692460646339759640 - T053328012456180694872856+10 .8155267119en90s354423560940 . 904689107550732sege131218*0 .966724794852572301596207440 997351236512808206195065040
$.2436805964941454829532664-1$ $.5538409304310284127512968-1$ .8566889948233679091726461-1 108599581274615567618499140 $1308762833968818041547260+0$ $1300762506018041547260+0$ $.1458456406304564155117133+0$ $.1682309488939117823945766+0$ .1871677878485580625475975+0 .209067911816311616926152940 .2385273341646520210799933+0 .2806846337671306774780527 t0 $.4593584320110012635634962+\square$
$.5429788413416968380732802-2$ $.2840068715574590750239410-1$ 6897995erazerar $.1254409556317280585382583+0$ 1960536143168552320171 595+0

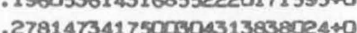
$.368694939 e 5335$ seccer $938416 * 0$ . $4643198423214430526570766+0$ . 5614230178214642328267213+0 $.6563228104863825961320366+0$ .745400520405286744389880040 .825244657148882272901461040 . $89 e 787404254140176780685740$ $9454271736998061753908600+0$ 9811319351210913389837062 $998505085989204636036171+0$

.24457109551078e22797453940-2 . $1284318750827285794015242-1$ $.3137358528490702374308480-1$ $.577422906212618954404887-1$ .9152265990831601977476978-1 $1321645365241536910985975+0$ $.1790018710891144679981008+0$ 231720093189558167509040 2907893796949333700347690 . 2800 rosorosentos . . 4115 . 4760785108ce1207416835926+C . 541076228314945745175550000 .60541508785354644Z2908734t0 $.6680012531107167367682701+0$ $.7277682874068713229591999+0$ 7836958884338745148098214 834827830306809439363982940 (1) (1) $.9754168352947288096399360+0$ .9915591635768725210049186+0 $.9993333998143269062485147+0$
$.1391103825270637716824364-1$ $3192635397215304550542838-1$ $489651726982529 e 964576903$ - 1 $.6455266493602585425443367-1$ $7885671385605241947004000-2$ $9164837942042515296430030-1$ $1032553877123435937031784+0$ . 11401650062791003700848000 .124355707074Z23882700167840 $.1347876812758415779436306+0$ $.1459745814246057647292903+0$ $1588761953193046161651780+0$ $1751391415493901301624227+0$ 196е8960 376 23000019935060905540519 3796250525706120723099011 to $N=24$

$6271585350916622504767417-2$ 1450 resiata $739 e 6749859482-1$ . $2252481411378850388273953-1$

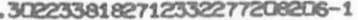
$.3752258634461099554661572-1$ .443781278Ce99266640101332-1 $5077643327871736729338775-1$ 5673142517374345405468185-1 c270055415178119249e377-1 . (1) (1) 7110031763657254169110365-1 . $8628459630064329265368239-1$ 9097747280793962475322563-1 $.9591935775547927605544475-1$ $10129 e 9537534091044138457+0$ $1073501607143330533277165+0$ $114468490409537017743457+0$ .123272209966547099265411440 .1349517518240564311497563+0 $1520740783040970258306163+0$ $.1828016156571662549162769+0$ $.29002327233486544098126234+0$ 
TABLE 13.- ERROR CONSTANTS $\mathrm{k}_{\mathrm{N}}$

\begin{tabular}{|c|c|c|c|c|c|c|c|}
\hline \multirow{2}{*}{$\begin{array}{c}\text { Weight } \\
\text { function }\end{array}$} & \multicolumn{7}{|c|}{$\mathrm{N}$} \\
\cline { 2 - 7 } & 2 & 4 & 6 & 8 & 12 & 16 & 24 \\
\hline 1 & $0.88323-2$ & $0.35333-4$ & $0.13925-6$ & $0.54651-9$ & $0.83793-14$ & $0.12817-18$ & $0.29917-28$ \\
2 & $.38568-2$ & $.15381-4$ & $.60574-7$ & $.23766-9$ & $.36431-14$ & $.55722-19$ & $.13006-28$ \\
3 & $.83723-2$ & $.33428-4$ & $.13195-6$ & $.51784-9$ & $.79396-14$ & $.12145-18$ & $.28347-28$ \\
4 & $.40962-2$ & $.16231-4$ & $.63926-7$ & $.25081-9$ & $.38488-14$ & $.58808-19$ & $.13726-28$ \\
5 & $.78509-2$ & $.31380-4$ & $.12366-6$ & $.48528-9$ & $.74402-14$ & $.11381-18$ & $.26564-28$ \\
6 & $.43400-2$ & $.17319-4$ & $.68214-7$ & $.26764-9$ & $.41029-14$ & $.62755-19$ & $.14647-28$ \\
7 & $.75454-2$ & $.30175-4$ & $.11891-6$ & $.46663-9$ & $.71543-14$ & $.10943-18$ & $.25543-28$ \\
8 & $.45125-2$ & $.18010-4$ & $.70939-7$ & $.27834-9$ & $.42669-14$ & $.65265-19$ & $.15233-28$ \\
9 & $.18611-1$ & $.72707-4$ & $.28401-6$ & $.11094-8$ & $.16925-13$ & $.25831-18$ & $.60142-28$ \\
10 & $.20227-2$ & $.79014-5$ & $.30865-7$ & $.12057-9$ & $.18397-14$ & $.28071-19$ & $.65359-29$ \\
11 & $.13386-1$ & $.51613-4$ & $.20089-6$ & $.78342-9$ & $.11935-13$ & $.18198-18$ & $.42341-28$ \\
12 & $.11944-1$ & $.46749-4$ & $.18286-6$ & $.71488-9$ & $.10918-13$ & $.16668-18$ & $.38828-28$ \\
\hline
\end{tabular}


TABLE 14.- THREE-TERM RECURRENCE RELATION PARAMETERS FOR WEIGHT FUNCTION 1

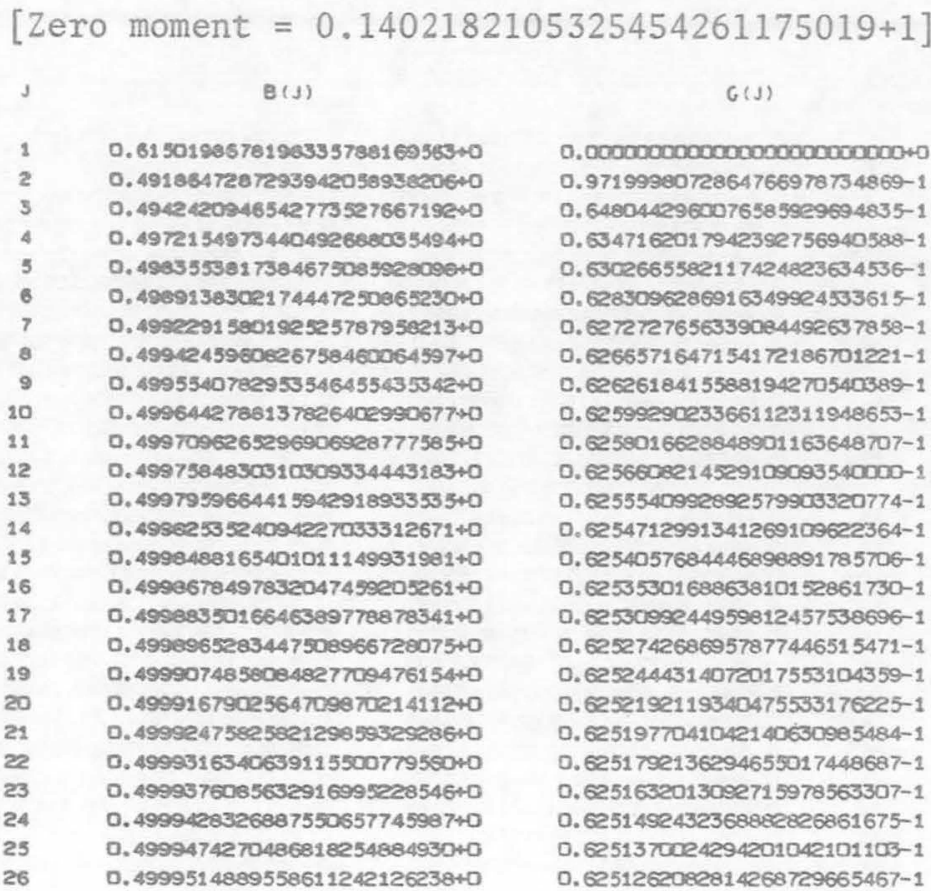

TABLE 15.- THREE-TERM RECURRENCE RELATION PARAMETERS FOR WEIGHT FUNCTION 2 $[$ Zero moment $=0.8413092631952725567050114+0]$

1

2

3

4

3

6

9

10

11

12

13

14

15

16

17

18

20

21

22

23

24
$B(j)$

$0.4392929058855954134405831 * 0$ $0.4352017100183943815546000 * 0$

0.49523407219646861 701 18784no

0.497559406178239831307990040

$0.49651369228864659030 a 901$ s:0

0.4990001 2393946478eser $4603 \% 0$

D. 499281385406560305867402640

$0.49245836329337825189 e 2525 \%$

$0.4995773992440698 e 0005717340$

0.499660977170195395591779440

0.499721930733151165054669740

$0.49976789 e 391108175206420840$ $0.49960325 e 3765095789489353 \div 0$ D. 499831167346735752009956340

$0.4998535090524275490334804+0$

$0.4998716911400500233698867+0$

0.499886685889119597551589740

0. $4998991971771692743253767 \div 0$

$0.4999097447421072889977161+0$

0. 49991871911605873062485694

0.4999264183447872010118851 to

$0.4999330730828293369672846+0$

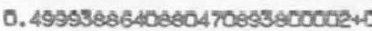

0.499943934637389310654455040

0.499948399481823069118748240

$0.4999523514007295296044060+\mathrm{C}$
$G(J)$

0. $0000000000000000000000000 \%$

0. 711541 5esosce $764271108506-1$

0. $6442813666042976091605560-1$

0. $6332966745806871531576270-1$

$0.6296973592602236653531604-1$

0. 628cR11458494534797182123-1

$0.6271063547685535342092320-1$

0. $265525449907410750921175-1$

0. ๓261918246049314627814951-1

D. $6259437591540021127428069-1$

0. $257658535834642161731121-1$

0. ₹2563392612231 42347617901-1

$0.6255333880575095221001711-1$

0. 6254550120226655985960952-1

0. €253927301393207138239993-1

$0.625342417502602321354261-1$

$0.62530119170474192243669 \mathbb{2}^{2}-1$

D. 6e52689886828711531437449-1

0. 6252382989725239067226655- 1

0. œ25213997993782e585789650-1

0. $6251932340168467508461636-1$

D. ₹51753523559262072777206-1

o. 6251 398431194931680803091-1

D. $6251463043927422864958548-1$

0. $6251344159158641393491053-1$

‥ ₹251239199535311867247599-1 
TABLE 16.- THREE-TERM RECURRENCE RELATION PARAMETERS FOR WEIGHT FUNCTION 3

$$
\text { [Zero moment }=0.1311028777146059905232420+1]
$$

4

2

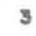

4

5
$B(J)$

0. 509070117367796105719961240 0.496575300391231306594127940 0.494206590223643390692613240 0.497250018634630970177733440 0.498368334706216340082588040 $0.4909807115762312343521780 \%$ $0.499233280 \mathrm{cos} 0389 \mathrm{~s} 9619131640$ 0.499427260731962454268722440 0.499535899912365042805876640 0.499645578314677471104087770 $0.4997105865356024598902937+0$ 0.499759211990766842308577940 $0.4997965329291087615448246+0$ 0.499825801331674556284820940 0.495949178301213752852490240 0.499868145561172878366753040 $0.4998837465770987898195050+0$ 0. 499896733416477647648937240 $0.4999076392322160168824781+\mathrm{C}$ $0.499910338228896046379858+0$ 0. $4999248855154766978370629 \times$ $0.49993174430 e 8827493188126+0$ 0. $4999377046879372664677897+0$ 0.499942917009168579412187240 $0.4999475014210552731992583+0$ 0.499951554885432493096091240
$G(J)$

$0.0000000000000000000000000 \% 0$ $0.9806157552139862646437001-1$ $0.6512306687296883760940316-1$ 0. $3345674644453224600076581-1$ 0. 63021 $66220438324487745683-1$ 0. 6282864671 434263341294804-1 0. $2772595745632631642853379-1$ 0. £266489347407277283216556-1 o. உ62563614141393223819131-1 D. $6259890703886055397170153-1$ $0.6257988789342640280374204-1$ 0. $256587355905226469287555-1$ 0. $255524963080244789721383-1$ 0. $€ 254700407730080482077893-1$ $0 . \approx 54047625922939490837249-1$ 0.œ5352200e612800804597432-1 0. $253092524613064958977411-1$ 0. ๓252737090370467398344471-1 0. $6252439604041707708777642-1$ 0. 2521881180874®4C2414351-1 0. 251973613204479311178697-1 $0 . £ 5178917734056942615230-1$ 0. $£ 51629441264089274169945-1$ o. $£ 251490182977750387040971-1$ 0. $\approx 51368045618503435808750-1$

๑. ๒51260333100e28893355157-1

TABLE 17.- THREE-TERM RECURRENCE RELATION PARAMETERS FOR WEIGHT FUNCTION 4 [Zero moment $=0.8740191847640399368216132+0]$

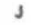

$B(J)$

$G(1)$

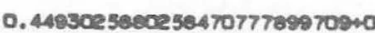
0.484447295041899089828946840 0.495249668249686313596771040 0.497531460126648474842410040 $0.48350 e 3 s e s e 9 e r 260007485840$ $0.4909240498814565716480631+0$ 0.498277642257828070669268940 0.499456112557136534252624040 0.499575707494535639763004740 $0.4996597605308907849517661+0$ 0.499721086663317039323433440 0.499767202348813676971452340 0. $4998 c e 753794764967867817640$ 0.499830738951502 roces 5339040 0.499853162724615808129097540 $0.4998714071878286934799331+\square$ $0.4998864501898053314500696+0$ $0.4996999993891209937789153 * 0$ 0. 499909577152177394808255940 0. 499918573876971254628475940 D. 49992e29495867613334774443+0 0. $4999329660441485916410501 \div 0$ 0.499938770532039640210121340 $0.4999438525599051594134650 \div 0$ 0. 499948327006262527668916940 0.499952287089381990778530440
0.000000000000000000000000000 $0.7229513301995411333776930-1$ $0.6439933981136391471174205-1$ $0.6333844246251481963630553-1$ $0.0297404143551978993652384-1$ 0. 6280415357384917522671346-1 0.6271 1823455699966339525942-1 $0.6265600092090025239833507-1$ 0. $261968906479339953848994-1$ $0 . £ 259473322475693390116272-1$ $0 . £ 57684674954987965057991-1$ 0. $656358957201063222902333-1$ $0 . £ 55349008761947173211291-1$ 0. ๓2545e2108885519244057762-1 0. $253936915837527579414533-1$ 0. $6253432004126267878853528-1$ D. $6253012376844604379888347-1$ $0 . £ 52675278900052877356511-1$ 0. $252387536969785377342962-1$ 0. $252143850006430134909680-1$ D. $6251935661164539603196196-1$ 0. $251756394597657275221424-1$ 0, (251600930023114625219419-1 o. $62514652322000624994069390-1$ D. 6251346086276909425429012 - 1 o. $251240905454199895408462-1$ 
TABLE 18.- THREE-TERM RECURRENCE RELATION PARAMETERS FOR WEIGHT FUNCTION 5

[Zero moment $=0.1214325323943790805909971+1]$

了

2

3

4

5

6

7

10

11
$B(J)$

$0.5773502691896257645091488+0$ o. $5073244852932461776984732+0$ 0. $4948795105676222733388612+0$ $0.4972672078130981410627300+0$ $0.4983825039421185072184619+0$ 0.498928296838042970251218940 0.4992378482546192918920371 t0 $0.4994302188104353046369573+0$ $0.4995579238210856127905567+0$ 0.499647023873442549608129240 0.4997116540121911954372261 +0 0.4997600227601140120087351 to $0.4997971631227829 e 8 c e 81298+0$ D. 499826300827589831848744940

$0.4998495808796588927641945+0$ $0.4998684747575800217293738+0$ 0. $4998840191953451561375046+0$ $0.4996969617132075425616461+0$ $0.4999078523170157899616100+0$ 0.4999171 cesecel $198047639034+0$ $0.49992502722 £ 579700092354+0$ 0. 499931867072633105702468240 $0.4999378117472204483070474+0$ $0.4999430109281509055388034+0$ 0.499947584265101121 CeOTC25+0 $0.499951 \Subset 283295563852513043+0$
$G(J)$

$0.0000000000000000000000000+0$ $0.9785159320496508915891917-1$ D. $6607151331035211590372786-1$ 0. $6342595664823952735501140-1$ $0.6301773597756086989783538-1$ D. £28606066997644894368888-1 0. $272450039424414981486837-1$ o. $\approx 266398039664500288805171-1$ o. ๒2ø5:2749104794835C220060. $\approx 59848117780434682202012-1$ 0. $257957838 c 22860350318375-1$ 0.๙56564159159946139222956-1 0. $£ 255507132375737305387282-1$ 0. $\approx 54686407717 £ 25597071578-1$ o. $254036433358142533188712-1$ o. $2253512914322700023485744-1$ $0.6253085044489808305010964-1$ o. $\approx 52730860304818867801960-1$ 0. $€ 252434360306568242235023-1$ 0. $252183662993729371625628-1$ 0. $25196790210921368302859-1$ 0. $๔ 51785882178947886573821-1$ 0. 凤251ø2657@86311138886451-1 0. $£ 5148767761 @ 24519194694-1$ 0. $\approx 51365841 \Subset 26843977796821-1$

$0 . \Subset 2125838401663907101 \approx 247-1$

TABLE 19.- THREE-TERM RECURRENCE RELATION PARAMETERS FOR WEIGHT FUNCTION 6

$$
\text { [Zero moment }=0.9107439929578431044324781+0]
$$

$$
B(J)
$$

$$
G(J)
$$

0.461880215351700611607319040 $0.4842922474383111053137346+0$ $0.4951303458690923598814542+0$ $0.4975060384373699610821943+0$ 0.498490145375790438430580040

$0.4989871213782979101558020+0$ $0.4992734249441331096310103+0$ $0.4994533523708811746413716+0$ $0.4995738038362481431348757+0$ 0.499658392653263148756146940 $0.4997200709624524781 \approx 21921$ to o. 499766427591741410088670540

$0.4998 c e 1494165093515891021+0$ $0.4998302584402616058791617+0$ 0.4998527744167714238694441 t0 $0.4998710889242144546537165+0$ 0.499886186087661515860109640

$0.4998987778240754602995538+0$ 0. $4999093894584215274819010+0$ 0,499918415487750161729274240 $0.4999261568245507204524516+0$

$0.4999328462309934921190331+0$

$0.4999386660380116782913450+0$

$0.4999437607127858799346190+0$

0. $4999482459181128238046202+0$

$0.4999522151399067760115839+0$
$0.0000000000000000000000000+0$ 0. $7412328435886561499483500-1$ $0.6428962495683730122573380-1$ $0.633 \approx 272305060683443434178-1$ 0. 6297744324108638830802400-1 o. $6280650351430805483073168-1$ 0. $271315883112505743978424-1$ 0. ๔265685484936632039630714-1 D. ๔e2ce5937055727058592352-1 0. $259513511232413999369233-1$ o. $257714052088745618903800-1$ 0. ஜ56381077933834168882223-1 0. $£ 25366159283641267640380-1$ D. $£ 54575556732714490780611-1$ 0. $253947697735688558167979-1$ 0. $253440780695731512164820-1$ o. ๒253c25616112264421992180-1 0. ๒52681319936344490558501-1 0. $2252392630303689392625671-1$ 0. $252148184005466529684303-1$ 0. 251939379440144269702956-1 0.£51759608535072589354035-1 0. $151 \lll 3726861702687955144-1$ o. ₹51467681103248427978876-1

0. $๒ 51348242644722062583708-1$

o. $251242814086083723663851-1$ 
TABLE 20.- THREE-TERM RECURRENCE RELATION PARAMETERS FOR WEIGHT FUNCTION 7

\begin{tabular}{|c|c|c|}
\hline J & $B(J)$ & $G(J)$ \\
\hline 1 & 0. $5633538790014043956734080+0$ & $0.0000000000000000000000000+0$ \\
\hline 2 & $0.5119583717209448584565940 \div 0$ & $0.9684596938716606430115540-1$ \\
\hline $\mathbf{3}$ & $0.4962540467307169696047499+0$ & $0.6695734049367672681943645-1$ \\
\hline 4 & $0.4972543007585147119961488+0$ & $0.646714493388579694323679-1$ \\
\hline 5 & 0.4983921392124710399060651 to & $0.6301213826076785229610760-1$ \\
\hline 6 & $0.498932548945049378 C 24964940$ & $0 . \Subset 284804237799797464737085-1$ \\
\hline 7 & $0.4992404466000627510249753+0$ & $0 . \notin 272365704741117522290901-1$ \\
\hline - & $0.4994319027130564147704560 \% 0$ & $0 . £ 266346179791909180770479-1$ \\
\hline 9 & 0.499559075932969582867396240 & $0 . £ 2 € 2468099577302312091489-1$ \\
\hline 10 & 0.499647846759631649509679240 & $0.6259823871111705993375620-1$ \\
\hline 21 & $0.4997122 ø 20389 e 25946160946+0$ & $0.1257940210634034684810536-1$ \\
\hline 12 & $0.4997604846756432403485743+0$ & $0.6256550944897801616402807-1$ \\
\hline 23 & 0.499797522233505121319547440 & 0. $255496972716809250477870-1$ \\
\hline 14 & $0.499 e 265855131419871021771+0$ & $0 . £ 54678429183523106012187-1$ \\
\hline 15 & $0.4996498103648121979900767+0$ & $0.6254030053675376051334807-1$ \\
\hline 16 & 0.499668662439486936394940940 & $0.6253507733264366281679306-1$ \\
\hline 17 & 0.499824174641209154921458740 & $0 . \Subset 253000779626968600244540-1$ \\
\hline 18 & $0.4996970919025462371016782+0$ & $0.6252727307731272198139451-1$ \\
\hline 19 & $0.4999079624381221807334044+0$ & $0 . £ 252431369830966108100039-1$ \\
\hline $\mathbf{\infty}$ & $0.4999171969546787304097069+0$ & $0 . \Subset 252181122015177250829386-1$ \\
\hline 21 & 0.499925108062666954075191740 & $0 . \approx 51967618970646698117800-1$ \\
\hline 2 & 0.49993193711 ce $442767946124 \div 0$ & $0.6251784002433503924641996-1$ \\
\hline 23 & $0.4999378728299778307367276+0$ & $0.6251624942911825352398175-1$ \\
\hline 24 & 0.499943064514833246640635040 & $0 . £ 5148 \propto 248200589612834210-1$ \\
\hline 25 & 0.499947631535915250654650740 & $0.6251364584069531914596666-1$ \\
\hline 26 & $0.49995167 \pi^{2} 393120999680997+0$ & $0 . £ 251257271835370214173250-$ \\
\hline
\end{tabular}

TABLE 21.- THREE-TERM RECURRENCE RELATION PARAMETERS FOR WEIGHT FUNCTION 8

$$
\text { [Zero moment }=0.9308740569746155002420145+0]
$$

J

$$
B(J)
$$

0.469461563834503663061174040 0.484952695013591213859399040 0.494977179722085155123407240 $0.4975175742671236633519 e 5440$ $0.49848032285205289 e 9148041$ to $0.4989033121879826349802523+0$ $0.4992709831252 \pi 0786105313040$ 0.499451762738184592966139040 $0.4995727087142081447920463+0$ 0.499657606308662996049981040 0.499719487444813710322368940 $0.4997659827178376178674056+0$

$0.4996018 c e 519491901501429940$ 0.499829982735128287893573340 0. 499852551681183454647741740 0.499870906412607885667856240 0.499886034668829524454078440 0.499698650817718107929060940 0. 499909281886225736327411540 0. 499918323578434147938824940 0.499926077679024517152140740 $0.4999327775910696071722080+0$ 0. $499938606123 \approx 253396626 \pi 0+0$ 0.499943708105479613877749740

0. 499948199476347450825361340

0. 499952173936638538647616940
$G(J)$

$0.0000000000000000000000000 \% 0$

$0.7547266847071244641551846-1$

$0.6423043428644020996393976-1$

$0.6336884753618849230806122-1$

$0.6296076834367451093252475-1$

0. $6280770657137456874476197-1$

0. $6271394724628407929925583-1$

0. $265734176943588756216890-1$

0. ๔262058778261776837657233-1

0. $659536622512632961448388-1$

0. $25773093424 \approx 212654360975-1$

0. $256393782938113460159507-1$

0. $255375959250571905342461-1$

$0 . \approx 254583274052183408159435-1$

$0.6253953883172798174534847-1$

$0 . £ 253445814328289731700786-1$

0. ๒25302976707602537165791-1

0. ๒252684783126716080379340-1

0. ๒5239554965716828201 5660-1

0. $6252150667689005253806993-1$

0. $251941510009904994294668-1$

0. $£ 51761449882180114920924-1$

0. $25165329055893731001556-1$

o. $251409083831203642491290-1$

0. $๒ 51349477690421812585691-1$

o. $£ 51243907146491366938 £ 26-1$ 


\begin{tabular}{|c|c|c|}
\hline J & $B(J)$ & $G(J)$ \\
\hline 1 & 0.431184926538298422492252540 & $0.0000000000000000000000000 \%$ \\
\hline 2 & $0.5344112677185342651850937+0$ & $0.1215894930360789821822357+0$ \\
\hline 3 & $0.4997081663357153245114400+0$ & $0.6302335181643522600332 \pi 03-1$ \\
\hline 4 & $0.4999979923236526420216647+0$ & $0.6250797750313006342035336-1$ \\
\hline 3 & $0.5000000753559464804948454+0$ & $0.6249996102798235805729757-1$ \\
\hline 6 & 0. $5000000013213312142794695+0$ & $0.6249999662216613204448769-1$ \\
\hline 7 & $0.4999999999586801854786577+0$ & $0 . \approx 250000000704277852836404-1$ \\
\hline 8 & $0.4999999999988 \approx 24260515453+0$ & $0.6250000000232057766949852-1$ \\
\hline 9 & $0.5000000000000277664504764+0$ & $0.6250000000000495634934922-1$ \\
\hline 10 & 0. $5000000000000010935031495+0$ & $0 . \approx 249999999999810836830959-1$ \\
\hline 12 & $0.4999999999999999800990423+0$ & 0. $6249999999999998709267165-1$ \\
\hline 12 & $0.4999999999999999988887928+0$ & $0.6250000000000000167223660-1$ \\
\hline 13 & $0.5000000000000000000140255+0$ & $0.1250000000000000001995067-1$ \\
\hline 14 & D. 500000000000000000001164040 & 0. $๔ 249999999999999999845983-1$ \\
\hline 15 & $0.4999999999999999999999913+0$ & 0. $๔ 49999999999999999997285-1$ \\
\hline 16 & 0.499999999999999999999998040 & $0.6250000000000000000000144-1$ \\
\hline 17 & 0. $5000000000000000000000000+0$ & $0.1250000000000000000000004-1$ \\
\hline 18 & 0. $5000000000000000000000000+0$ & $0.6250000000000000000000000-1$ \\
\hline 19 & 0. $5000000000000000000000000+0$ & $0.6250000000000000000000000-1$ \\
\hline 20 & 0. $5000000000000000000000000 * 0$ & $0.6250000000000000000000000-1$ \\
\hline 21 & 0. $5000000000000000000000000+0$ & $0 . \approx 250000000000000000000000-1$ \\
\hline 22 & $0.5000000000000000000000000+0$ & $0 . £ 50000000000000000000000-1$ \\
\hline 23 & 0. $5000000000000000000000000+0$ & $0.6250000000000000000000000-1$ \\
\hline 24 & c. $5000000000000000000000000+0$ & $0 . \approx 250000000000000000000000-1$ \\
\hline 25 & 0. $5000000000000000000000000+0$ & $0.6250000000000000000000000-1$ \\
\hline 26 & $0.5000000000000000000000000+0$ & $0.6250000000000000000000000-1$ \\
\hline
\end{tabular}

TABLE 23.- THREE-TERM RECURRENCE RELATION PARAMETERS FOR WEIGHT FUNCTION 10

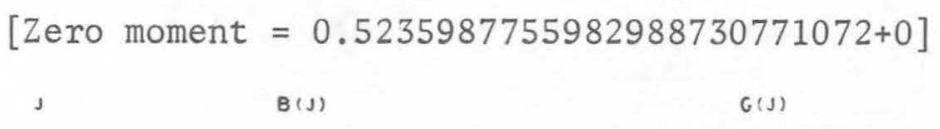

$10.5348805958594948912557218 \% 0$

$20.4998413758642581247934532+0$

$30.4999755312512212612701437+0$

$40.3000000431490767513877482+0$

5 0. 500000024285702978672929840

$6 \quad 0.5000000000653895318792445+0$

$7 \quad 0.4999999999739740331698369+0$

B $0.499999999999803 \mathrm{T01} 4320393+0$

$90.5000000000000285940367426+0$

$10 \quad 0.5000000000000003647314906+0$

$110.4999999999999999684488097+0$

$12 \quad 0.4999999999999999994158492+0$

13 0. $5000000000000000000345507+0$

$140.5000000000000000000008687+0$

15 0. $4999999999999999999999628+0$

$160.4999999999999999999999980+0$

$17 \quad 0.5000000000000000000000000+0$

$180.5000000000000000000000000+0$

19 0. $5000000000000000000000000+0$

20 $\quad$ D. $5000000000000000000000000+0$

$21 \quad 0.5000000000000000000000000+0$

$220.5000000000000000000000000+0$

$230.5000000000000000000000000+0$

24 0. $5000000000000000000000000+0$

25 0. $5000000000000000000000000+0$

$260.5000000000000000000000000+0$
$0.0000000000000000000000000+0$

$0.617813282 \propto 217020632362457-1$

0. 252901993772103984020583-1

0. $6250068874693278612242355-1$

o. $249997553471393707650941-1$

0. $\approx 249999918983040738478722-1$

o. $\approx 50000002234182735731698-1$

o. $250000000099618865540936-1$

0. $\approx 249999999997963060999185-1$

0. $\approx 249999999999876211407871-1$

0. $6250000000000001758908590-1$

o. $250000000000000153639510-1$

0. $\approx 249999999999999998677223-1$

0. ₹249999999999999999810552-1

0. $\approx 50000000000000000000645-1$

0. $1250000000000000000000231-1$

0. $1250000000000000000000000-1$

0. $650000000000000000000000-1$

0. $\approx 50000000000000000000000-1$

0. $250000000000000000000000-1$

0. $£ 50000000000000000000000-1$

0. $150000000000000000000000-1$

0. $6250000000000000000000000-1$

0. $1250000000000000000000000-1$

0. $1250000000000000000000000-1$

$0.1250000000000000000000000-1$ 
TABLE 24.- THREE-TERM RECURRENCE RELATION PARAMETERS FOR WEIGHT FUNCTION 11 [Zero moment $=0.2622057554292119810464840+1]$

$B(1)$
0. $7627597635018131880623260+0$
o. $4356406220177258406970294+0$
0. 483662225381225795750420440
o. $4926359637757834902129061+0$
0. $4958160622403241212889954+0$
$0.4973025753683422193594969+0$
0. $4981164296636529946910406+0$
0. $4986102355901269385187147+0$
0. $4989323521912857650120867+0$
0. $4991541052638607539612490+0$
0. $4993132670282797887930142+0$
0. $4994313681 \Subset 29815576863545+0$
$0.4995214144758561080843496+0$
D. $4995916401095205920752087+0$
o. $4996474647387156655116376+0$
D. $4996925741142250124147184+0$
0. $4997295457223993006853085+0$
0. 499760225997832135725549940
$0.4997859657879120033126338+0$
$0.4998077718433315107473383+0$
$0.499826406901 \approx 278370299617+0$
0. $4998424573786985573892202+0$
0. $4998563801202517857375893+0$
$0.4998685353030452 \approx 2 \pi 08292240$
0.4998792099848722599216561 to
$0.4998886352223224496996331+0$

$G(J)$

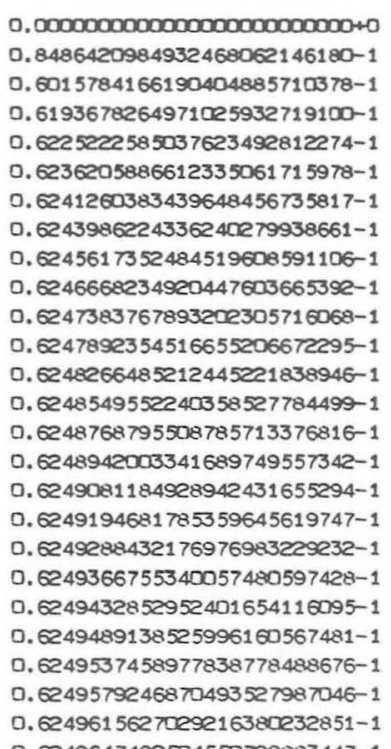

$0.0000000000000000000000000+0$

$0.84864209849324680 £ 2146180-1$

0. ๔225222585037623492812274-1

o. ®3ø20588661233506171 5978-1

0. $\propto 241260383439648456735817-1$

o. $246668234920447603665392-1$

$0 . £ 24383767893202305716068-1$

0. ๓47892354516655206672295-

0. $248768795508785713376816-1$

$0 . £ 248942003341689749557342-1$

0. $\circledast 249081184928942431655294-1$

0. $249366755340057480597428-1$

249432852952401654110095-1

$0.6249537458977838778488676-1$

จ. $\approx 4961562$ TL2921 638C232851-1

0. $249647492504550799883447-1$

TABLE 25. - THREE-TERM RECURRENCE RELATION PARAMETERS FOR WEIGHT FUNCTION 12

\section{$[$ Zero moment $=0.2103273157988181391762529+1]$ \\ J \\ $B(J)$ \\ $G(J)$}

0. 713174127812659855007629140 $0.4574649756003002978073342+0$ $0.4880003168683295789202167+0$ o. $4944457136921354886874163+0$ 0. $4968059789822665620923860+0$ 0. $4979263010407050897093034+0$ $0.4985452766986458567019637+0$ 0. $4989231367944321599964818+0$ $0.4991707046055156317255353+0$ 0. $4993417030237524201212730+0$ 0. $4994647555958287820432594+0$ 0. $4995562544 \approx 24473435839419+0$ $0.4996261382321980163249037+0$ $0.4996807182051692635412360+0$ 0. $4997241588988035784313898+0$ 0. $4997592984903970030365944+0$ 0. $4997281253 \Subset 24322011219338+0$ 0. $49981206618035027 \approx 2728449+0$ 0. $4998321661305429612525275+0$ 0. $4998492051120634935611432+0$ 0. $4998637746348907397280967+0$ $0.4998763299112804176981203+0$ 0. $49988722588304289 e 2387086+0$ o. $4998967426074243971984465+0$ 0. $4999051034483297284078400+0$ $0.4999124883125537396555600+0$
$0.0000000000000000000000000+0$ 0. $9138266341865190505355904-1$ D. 凤14157546585639457435741-1 0. $£ 56266089751222097592868-1$ 0. $£ 56299807035465916956908-1$ 0. $254803891898191389681332-1$ 0. $® 25641066555803461296587-1$ 0. $๒ 252821015902222334908550-1$ 0. ๒52238768292636176ø28142-1 0. $1251815423805393280826464-1$ 0. $£ 51499729997178538489978-1$ 0. $251258760325053868405053-1$ 0. $1251070987590363060099149-1$ 0. $£ 250921991884649904453897-1$ 0. $๒ 250801871899012795326180-1$ o. $150703668503265348254934-1$ 0. $\approx 50622384898620495766689-1$ 0. $250554363219312863216430-1$ o. $250496878593744372225568-1$ o. $250447868911064312231361-1$ 0. $\approx 50405751193489093328913-1$ 0. $250369294431442926087396-1$ o. $\approx 250337529972380990634016-1$ 0. $250309687354327723032198-1$ 0. ๒50285147678126226373071-1

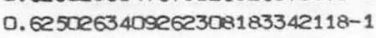




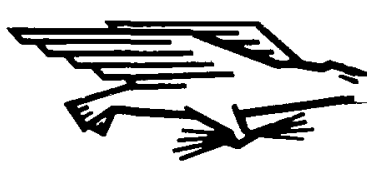

POSTAGE AND FEES PAID NATIONAL AERONAUTICS Ai SPACE ADMINISTRATION

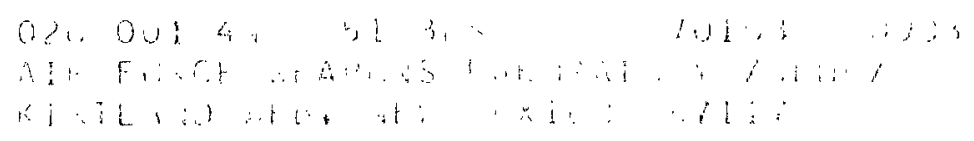

"The aeronantical and space activities of the United States shall be conducted so as to contribute... to the expansion of buman knowledge of phenomena in the atmosphere and space. The Administration sball provide for the widest practicable and appropriate dissemination of information concerning its actirities and the results thereof."

- National Aeronautics And Space Act of 1958

\section{NASA SCIENTIFIC AND TECHNICAL PUBLICATIONS $\because$}

TECHNICAL REPORTS: Scientific and technical information considered important, complete, and a lasting contribution to existing knowledge.

TECHNICAL NOTES: Information less broad in scope but nevertheless of importance as a contribution to existing knowledge.

TECHNICAL MEMORANDUMS:

Information receiving limited distribution because of preliminary data, security classification, or other reasons.

CONTRACTOR REPORTS: Scientific and technical informatiơn generated under a NASA contract or grant and considered an important contribution to existing knowledge.
TECHNICAL TRANSLATIONS: Information published in a foreign language considered to merit NASA distribution in English.

SPECIAL PUBLICATIONS: Information derived from or of value to NASA activities. Publications include conference proceedings, monographs, data compilations, handbooks, sourcebooks, and special bibliographies.

TECHNOLOGY UTILIZATION

PUBLICATIONS: Information on technology used by NASA that may be of particular interest in commercial and other non-aerospace - applications. Publications include Tech Briefs, Technology Utilization Reports and Notes, and Technology Surveys.

Details on the availability of these publications may be obtained from:

\section{SCIENTIFIC AND TECHNICAL INFORMATION DIVISION}

